\title{
Transport theory for disordered multiple-band systems: Anomalous Hall effect and anisotropic magnetoresistance
}

\author{
Alexey A. Kovalev, ${ }^{1,2}$ Yaroslav Tserkovnyak, ${ }^{1}$ Karel Výborný, ${ }^{3}$ and Jairo Sinova ${ }^{2,3}$ \\ ${ }^{1}$ Department of Physics and Astronomy, University of California, Los Angeles, California 90095, USA \\ ${ }^{2}$ Department of Physics, Texas A\&M University, College Station, Texas 77843-4242, USA \\ ${ }^{3}$ Institute of Physics, ASCR, Cukrovarnická 10, 16253 Praha 6, Czech Republic \\ (Received 16 February 2009; revised manuscript received 26 April 2009; published 28 May 2009)
}

\begin{abstract}
We present a study of transport in multiple-band noninteracting Fermi metallic systems based on the Keldysh formalism and the self-consistent $T$-matrix approximation (TMA) taking into account the effects of Berry curvature due to spin-orbit coupling. We apply this formalism to a Rashba two-dimensional electron-gas ferromagnet and calculate the anomalous Hall effect (AHE) and anisotropic magnetoresistance (AMR). The numerical calculations of the AHE reproduce analytical results in the metallic regime revealing the crossover between the skew-scattering mechanism dominating in the clean systems and intrinsic mechanism dominating in the moderately dirty systems. As we increase the disorder further, the AHE starts to diminish due to the spectral broadening of the quasiparticles. Although for certain parameters this reduction of the AHE can be approximated as $\sigma_{x y} \sim \sigma_{x x}^{\varphi}$, with $\varphi$ varying around 1.6, this is found not to be true in general as $\sigma_{x y}$ can go through a change in sign as a function of disorder strength in some cases. Furthermore, the disordered region consistent with the TMA is relatively narrow and a theory with a wider range of applicability in strong disorder limit is called for. By considering the higher order skew-scattering processes, we resolve some discrepancies between the AHE results obtained by using the Keldysh, Kubo, and Boltzmann approaches. We also show that similar higher order processes are important for the AMR when the nonvertex and vertex parts cancel each other. We calculate the AMR in anisotropic systems properly taking into account the anisotropy of the nonequilibrium distribution function. These calculations confirm recent findings on the unreliability of common approximations to the Boltzmann equation.
\end{abstract}

DOI: $10.1103 /$ PhysRevB.79.195129

PACS number(s): 72.15.Eb, 72.20.Dp, 72.20.My, 72.25.-b

\section{INTRODUCTION}

Recently, the interest in transport calculations in multipleband systems ${ }^{1,2}$ has been rekindled in part due to the realization of diluted magnetic semiconductors (DMSs) that have strong spin-orbit interactions, variable carrier densities, and ferromagnetic ordering. These properties imply the existence of the anomalous Hall effect (AHE) (Ref. 3) and the anisotropic magnetoresistance (AMR). ${ }^{4}$ Even though the mechanisms of the AHE and the AMR are different, they both have a similar description based on the multiple-band transport theory. In this paper, we formulate a relatively simple framework for doing such transport calculations.

The AHE is usually described in terms of the anomalous Hall resistivity $\rho_{x y}$ that measures the transverse voltage with respect to the transport direction and depends on the spontaneous magnetization $M$ along the $z$ direction. Theoretical studies of the AHE have a long history beginning with the work of Karplus and Luttinger. ${ }^{5}$ A number of papers on the AHE also appeared not so long ago ${ }^{6-12}$ after the interpretation of the AHE based on the Berry phase ${ }^{13}$ was proposed. Nevertheless, theoretical description of the AHE is far from being complete and it often involves cumbersome calculations without transparent interpretations. ${ }^{14}$ The difficulties appear due to the necessity to consider the off-diagonal elements in Bloch band indices (the interband coherences induced by charge currents). There is a general trend to focus on particular simple models in order to overcome the common mistakes that are made in treating the AHE. A number of recent publications concentrate on the simpler but non- trivial Rashba two-dimensional (2D) electron system, ${ }^{1,15-23}$ yet arriving at contradictory predictions. Most of the disagreements have been finally resolved ${ }^{22-24}$ with some being addressed in this paper.

In calculating the AHE for a given material, the usual approximations performed to leading order in $\hbar / \tau \varepsilon_{F}$ can fail, where $\tau$ is the scattering time and $\varepsilon_{F}$ is the Fermi energy. The semiclassical description of the Hall conductivity within the usual Boltzmann equation leads to an AHE contribution due to the scattering asymmetry in the collision term usually labeled as skew scattering. ${ }^{25}$ Other terms, arising from subtle issues dealing with interband coherence during the collision and acceleration by the electric field between collisions, are usually introduced by hand through the so-called anomalous velocity $^{26}$ and side jump. ${ }^{27}$ This approach however, is nonsystematic and prone to errors from missing terms and wrong interpretations, e.g., giving physical meaning to gauge dependent quantities. A more systematic way to derive the correct semiclassical equations is through the Keldysh formalism in which these interband coherence effects are taken into account automatically. ${ }^{1,24}$

The system under consideration also allows us to study the diagonal resistance as a function of the direction of the magnetization. The change in the resistance as a function of the magnetization direction relative to the current or crystallographic direction is called the AMR effect. The microscopic origin of the AMR in transition-metal ferromagnets is still elusive $\mathrm{e}^{28-31}$ and detailed calculations require consideration of complicated band structures. ${ }^{32,33}$ A relatively simple host band structure in the DMS ferromagnets provides a pos- 
sibility for performing detailed microscopic calculations based on simple physical models. ${ }^{34}$ However, the relaxationtime approximation used in such calculations is not always reliable since it does not fully take into account the anisotropies of the system. ${ }^{35}$ The Kubo formula approach has been applied to the AMR calculations in Rashba systems and it has revealed the cancellation of the nonvertex and vertex parts, ${ }^{36}$ similar to the spin Hall effect (SHE) and the AHE.

In this paper, we apply the Keldysh formalism for transport calculations in multiple-band noninteracting Fermi systems. This treatment simultaneously takes into account the Berry curvature effects (interband coherences) and scattering, allowing us to immediately account for such physical effects as side-jump scattering and skew scattering within the same footing. We calculate the AHE analytically and numerically for the Rashba model and find, in agreement with Onoda et al., ${ }^{1,20}$ three distinct regimes: the skew-scattering regime, the disorder independent regime, and the dirty regime in which, although the basis of theory is not as well established, a distinct rapid reduction of the AHE is observed as the conductivity $\sigma_{x x}$ diminishes. Even though almost all ferromagnetic systems are three dimensional (3D), the findings of this simple 2D model has been linked to higher dimensional systems arguing that most likely the major contributions to the AHE come from the band anticrossing regions ${ }^{1}$ similar to one observed in the Rashba model. We further analyze the scaling found in the dirty regime ${ }^{1,20}$ in which the AHE seems to diminish in a manner that can be approximated as $\sigma_{x y} \sim \sigma_{x x}^{\varphi}$, with $\varphi$ being close to 1.6. Some experimental results claim to confirm such scaling; ${ }^{37-41}$ however, treatment of some of these experimental results has to be done with extra care as the region of interest is often restricted to less than a single decade, the materials have strong mangetoresistances and in-plane anisotropies associated with them, and most of the data associated with the zero-field calculation are in fact at very high magnetic fields.

Although at first sight our numerical results may seem to confirm this scaling, the closer analysis reveals that the selfconsistent $T$-matrix approximation (TMA), which is the cornerstone of the formalism, fails when $\tau \varepsilon_{F} \sim 1$ leaving us with insufficiently wide range of applicability of our theory (and others based on the TMA) for scaling claims. In addition, for the repulsive impurity potentials, the crossover from the disorder independent (intrinsic) regime to the skew-scattering regime is always accompanied by the sign change of the AHE which can shrink the AHE reduction region even further. Although this simple model seems to capture qualitative aspects of the three regions, to make a quantitative link to $3 \mathrm{D}$ materials with much more complex behavior seems premature at this stage. In our calculations, we also identify the hybrid skew-scattering regime of the AHE (Ref. 24) resulting from the higher order scattering processes. We take such processes into account in our AMR calculations and conclude their importance for the Rashba model in which nonvertex and vertex diagrammatic parts can cancel each other. ${ }^{36}$ Our results suggest that the relaxation-time approximation is not always reliable for the AMR calculations as it has been shown recently within the Boltzmann equation treatment. ${ }^{35}$

The paper is organized as follows. In Sec. II, we develop a general formulation of transport in multiple-band noninter- acting Fermi systems with further generalizations in Appendix A. In Sec. III, we calculate the AHE in two-dimensional electron-gas (2DEG) ferromagnet with spin-orbit interaction. The analytical and numerical results are followed by discussions and comparison to other works. In Sec. IV, we calculate the AMR in 2DEG ferromagnet with spin-orbit interaction. Finally in Sec. V, we present our conclusions.

\section{TRANSPORT IN MULTIPLE-BAND SYSTEMS}

The method presented in this section can be applied to a multiple-band system described by a Hamiltonian $\hat{H}_{0}+\hat{V}(\mathbf{r})$ that is a matrix in the band (chiral) index. In this section, we first derive general nonlinear equations using nonequilibrium diagrammatic technique, further restricting our consideration to a linear-response theory.

\section{A. Quantum kinetic equation}

We start by defining the following Green's functions: ${ }^{42}$

$$
\begin{gathered}
\hat{G}_{11} \equiv-i\left\langle T_{c} \boldsymbol{\Psi}\left(1_{+}\right) \boldsymbol{\Psi}^{\dagger}\left(1_{+}^{\prime}\right)\right\rangle=-i\left\langle\vec{T} \boldsymbol{\Psi}\left(1_{+}\right) \boldsymbol{\Psi}^{\dagger}\left(1_{+}^{\prime}\right)\right\rangle, \\
\hat{G}_{21} \equiv-i\left\langle T_{c} \boldsymbol{\Psi}\left(1_{-}\right) \boldsymbol{\Psi}^{\dagger}\left(1_{+}^{\prime}\right)\right\rangle=-i\left\langle\boldsymbol{\Psi}\left(1_{-}\right) \boldsymbol{\Psi}^{\dagger}\left(1_{+}^{\prime}\right)\right\rangle, \\
\hat{G}_{12} \equiv-i\left\langle T_{c} \boldsymbol{\Psi}\left(1_{+}\right) \boldsymbol{\Psi}^{\dagger}\left(1_{-}^{\prime}\right)\right\rangle=i\left\langle\boldsymbol{\Psi}^{\dagger}\left(1_{-}^{\prime}\right) \boldsymbol{\Psi}\left(1_{+}\right)\right\rangle, \\
\hat{G}_{22} \equiv-i\left\langle T_{c} \boldsymbol{\Psi}\left(1_{-}\right) \boldsymbol{\Psi}^{\dagger}\left(1_{-}^{\prime}\right)\right\rangle=-i\left\langle\overleftarrow{T} \boldsymbol{\Psi}\left(1_{-}\right) \boldsymbol{\Psi}^{\dagger}\left(1_{-}^{\prime}\right)\right\rangle,
\end{gathered}
$$

where $T_{c}$ is the generalized time-ordering operator acting on the Keldysh contour which can be split in two time axes $t_{+}$ (forward) and $t_{-}$(backward), $\boldsymbol{\Psi}$ is the vector in the band (chiral) space corresponding to the Fermi field, and $1_{ \pm}$ $=\left(\mathbf{r}, t_{ \pm}\right)$is the variable that describes the spatial variable $\mathbf{r}$ and the time variable $t$. The generalized time-ordering operator performs an ordinary time ordering $\vec{T}$ for the time $t_{+}$, an antitime ordering $\overleftarrow{T}$ for the time $t_{-}$, and in the mixed case $t_{-}$ occurs always after $t_{+}$within the Keldysh time contour. We can now define the Green's function in the Keldysh space

$$
\tilde{G}=\left(\begin{array}{ll}
\hat{G}_{11} & \hat{G}_{12} \\
\hat{G}_{21} & \hat{G}_{22}
\end{array}\right) .
$$

The scattering potential due to impurities in the Keldysh space has the form

$$
\tilde{V}\left(1,1^{\prime}\right)=\left(\begin{array}{cc}
\hat{V}(\mathbf{r}) & 0 \\
0 & -\hat{V}(\mathbf{r})
\end{array}\right) \delta\left(1-1^{\prime}\right),
$$

where $\hat{V}(\mathbf{r})$ describes the potential in the band (chiral) space formed by many scatterers which for current consideration can have any general matrix form. The negative sign arises here simply because the lower branch integration is taken from $+\infty$ to $-\infty$ while in the Keldysh loop the time goes from $-\infty$ to $+\infty$. The Green's function in Eq. (2) allows for a perturbation expansion relying on the Feynman rules. However, the four matrix elements of a so defined Green's function are 
linearly dependent, i.e., $\hat{G}_{12}+\hat{G}_{21}=\hat{G}_{11}+\hat{G}_{22}$. Hence it is advantageous to perform a linear transformation in the Keldysh space to eliminate one matrix element in Eq. (2)

$$
\check{G}=\left(\begin{array}{cc}
1 & 0 \\
1 & -1
\end{array}\right)\left(\begin{array}{ll}
\hat{G}_{11} & \hat{G}_{12} \\
\hat{G}_{21} & \hat{G}_{22}
\end{array}\right)\left(\begin{array}{cc}
1 & 0 \\
-1 & 1
\end{array}\right)=\left(\begin{array}{cc}
\hat{G}^{R} & \hat{G}^{<} \\
0 & \hat{G}^{A}
\end{array}\right),
$$

which leads to the following scattering potential:

$$
\check{V}=\left(\begin{array}{ll}
1 & 0 \\
1 & 1
\end{array}\right) \tilde{V}\left(\begin{array}{cc}
1 & 0 \\
1 & -1
\end{array}\right)=\left(\begin{array}{ll}
1 & 0 \\
0 & 1
\end{array}\right) \hat{V}(\mathbf{r}) \delta\left(1-1^{\prime}\right),
$$

where $\hat{G}^{R}=\hat{G}_{11}-\hat{G}_{12}$ is the retarded Green's function, $\hat{G}^{A}$ $=\hat{G}_{12}-\hat{G}_{22}$ is the advanced Green's function, and $\hat{G}^{<}=\hat{G}_{12}$. There are other choices for the linear transformation and our choice is dictated by the fact that the Green's function $\hat{G}^{<}$ can be immediately related to the distribution function in the Boltzmann equation. ${ }^{43}$

As of now, it is assumed that $\hat{V}(\mathbf{r})$ describes some disordered potentials and all Green's functions are averaged over this disorder. In the transformed Keldysh space, the Dyson equation ${ }^{42}$ becomes

$$
\left(\begin{array}{cc}
\hat{G}_{0}^{-1}-\hat{\Sigma}^{R} & -\hat{\Sigma}^{<} \\
0 & \hat{G}_{0}^{-1}-\hat{\Sigma}^{A}
\end{array}\right) \otimes\left(\begin{array}{cc}
\hat{G}^{R} & \hat{G}^{<} \\
0 & \hat{G}^{A}
\end{array}\right)=\check{1},
$$

where $R, A$, and $<$, respectively, stand for the retarded, advanced, and lesser components of the disorder averaged Green's functions and self-energies. The symbol $\otimes$ denotes a convolution (in position, time, and band/spin). The diagonal components of Eq. (4) yield the two equations for the retarded and advanced Green's functions

$$
\left(\hat{G}_{0}^{-1}-\hat{\Sigma}^{R, A}\right) \otimes \hat{G}^{R, A}=\hat{1} .
$$

The off-diagonal component of Eq. (4) yields the kinetic equation (sometimes called quantum Boltzmann equation) which contains the nonequilibrium information necessary to study transport

$$
\left[\hat{G}^{R}\right]^{-1} \otimes \hat{G}^{<}-\hat{\Sigma}^{<} \otimes \hat{G}^{A}=0 .
$$

In order to solve Eq. (6), one has to calculate the selfenergy $\hat{\Sigma}^{<}$of the particular problem. Here we focus on scattering by randomly distributed identical impurities at zero temperature with

$$
\hat{V}(\mathbf{r})=\sum_{i} \hat{\eta} U\left(\mathbf{r}-\mathbf{r}_{i}\right),
$$

where $\mathbf{r}_{i}$ describes the positions of random impurities of density $n_{i}$ and $\hat{\eta}$ is some matrix in the band index (e.g., in Sec. III, it is a unit matrix corresponding to scalar impurities and in Sec. IV, it is a combination of unit and Hermite matrices corresponding to charged and magnetic impurities). A common approximation to this problem is the self-consistent TMA which takes into account all the noncrossing scattering events from single impurities (see Fig. 1). We assume here that the system is uniform and $\check{G}$ depends on the difference of spatial variables $\left(\mathbf{r}-\mathbf{r}^{\prime}\right)$ (however, this requirement can be

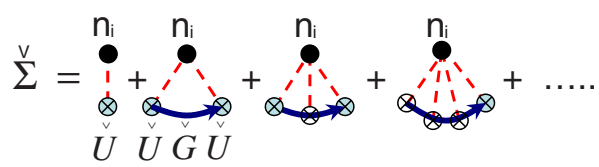

FIG. 1. (Color online) The nonequilibrium self-energy calculated using the self-consistent $T$-matrix approximation in Keldysh space.

lifted for the short-range disorder as it is shown in Appendix A). In this case, we can sum up the infinite series of diagrams in Fig. 1 arriving at the following expression for the selfenergy in the momentum representation (for the sake of compact form we use the momentum representation here):

$$
\left\langle\mathbf{k}|\check{\check{L}}| \mathbf{k}^{\prime}\right\rangle=n_{i}\langle\mathbf{k}|\check{T}| \mathbf{k}\rangle \delta\left(\mathbf{k}-\mathbf{k}^{\prime}\right),
$$

with the following expression for the $T$-matrix operator of impurity placed in the origin:

$$
\check{T} \equiv(\check{V}+\check{V} \otimes \check{G} \otimes \check{V}+\cdots)
$$

where $\check{V}=\left(\begin{array}{ll}\hat{\eta} & 0 \\ 0 & \hat{\eta}\end{array}\right) U(\mathbf{r}) \delta\left(1-1^{\prime}\right)$. Combining the $T$-matrix structure $\check{T}=\check{V} \otimes[1+\check{G} \otimes \check{T}]$ and solving for the off-diagonal component we obtain the equation for the lesser component of self-energy

$$
\left\langle\mathbf{k}\left|\hat{\Sigma}^{<}\right| \mathbf{k}^{\prime}\right\rangle=n_{i}\left\langle\mathbf{k}\left|\hat{T}^{R} \otimes \hat{G}^{<} \otimes \hat{T}^{A}\right| \mathbf{k}\right\rangle \delta\left(\mathbf{k}-\mathbf{k}^{\prime}\right) .
$$

The retarded and advanced $T$ matrices are given by the usual form

$$
\hat{T}^{R, A}=\hat{V} \otimes\left(1+\hat{G}^{R, A} \otimes \hat{T}^{R, A}\right)=\left(1+\hat{T}^{R, A} \otimes \hat{G}^{R, A}\right) \otimes \hat{V} .
$$

Equations (6) and (10) form a general closed set of equations for $\hat{G}^{<}$. In order to solve these equations, we can further simplify them by looking for a solution of the form

$$
\hat{G}^{<}=\hat{G}_{2}^{<}+\hat{G}_{1}^{<},
$$

where

$$
\hat{G}_{2}^{<}=n_{F} \otimes \hat{G}^{A}-\hat{G}^{R} \otimes n_{F}
$$

and the operator $n_{F}$ is the Fermi distribution function. In the case of zero temperature, $n_{F}$ is the step function in the frequency representation $n_{F}(\omega)=\theta(-\omega)$ and $n_{F}\left(t, t^{\prime}\right)=i /[2 \pi(t$ $\left.\left.-t^{\prime}+i 0\right)\right]$ in the time representation. Equations (12) and (13) will allow us to separate the Fermi sea and Fermi-surface components of the lesser Green's function. By substituting Eq. (12) into Eq. (6), we obtain the kinetic equation for $\hat{G}_{1}^{<}$,

$$
\left[\hat{G}^{R}\right]^{-1} \otimes \hat{G}_{1}^{<}-\hat{\Sigma}_{1}^{<} \otimes \hat{G}^{A}=\left[\hat{H}_{0}^{\otimes}, n_{F}\right] \otimes \hat{G}^{A},
$$

with $\quad\left\langle\mathbf{k}\left|\hat{\Sigma}_{1}^{<}\right| \mathbf{k}^{\prime}\right\rangle=n_{i}\left\langle\mathbf{k}\left|\hat{T}^{R} \otimes \hat{G}_{1}^{<} \otimes \hat{T}^{A}\right| \mathbf{k}\right\rangle \delta\left(\mathbf{k}-\mathbf{k}^{\prime}\right), \quad$ where $[\ldots, . .$.$] stands for a commutator. In order to derive Eq. (14),$ Eqs. (5) and (11) are used along with the fact that $\hat{T}^{R} \otimes \hat{G}_{2}^{<}$ $\otimes \hat{T}^{A}=n_{F} \otimes \hat{T}^{A}-\hat{T}^{R} \otimes n_{F}$ and

$$
\hat{\Sigma}_{2}^{<}=n_{F} \otimes \hat{\Sigma}^{A}-\hat{\Sigma}^{R} \otimes n_{F},
$$

which is a consequence of Eqs. (10), (11), and (13). 
The lesser Green's function contains all the information about the transport properties of our system and the charge current density can be calculated as

$$
\begin{aligned}
j_{x(y, z)} & =\frac{e}{2} \operatorname{Tr}\left\langle\boldsymbol{\Psi}^{\dagger}\left(1^{\prime}\right) \hat{v}_{x(y, z)}(1) \boldsymbol{\Psi}(1)\right\rangle_{1=1^{\prime}}+\text { c.c. } \\
& =-\frac{i e}{2} \operatorname{Tr}\left\{\left[\hat{v}_{x(y, z)}(1)+\hat{v}_{x(y, z)}^{\dagger}\left(1^{\prime}\right)\right] \hat{G}^{<}\left(1,1^{\prime}\right)\right\}_{1=1^{\prime}},
\end{aligned}
$$

where $\quad \hat{\boldsymbol{v}}(1)=\left(-i \hbar \boldsymbol{\nabla}_{1}-e \hat{\mathcal{A}}(1) / c\right) / m, \quad \hat{\boldsymbol{v}}^{\dagger}\left(1^{\prime}\right)=\left(i \hbar \boldsymbol{\nabla}_{1^{\prime}}\right.$ $\left.-e \hat{\mathcal{A}}^{\dagger}\left(1^{\prime}\right) / c\right) / m$, and $\hat{\mathcal{A}}(1)$ is the generalized vector potential matrix in the band index that also describes spin-orbit interactions; $e=-|e|$ stands for an electron charge.

\section{B. Linearized Fermi-surface contribution}

The kinetic Eq. (14) has not assumed linearity in electric field strength nor any particular temporal dependence. Higher order terms in the impurity density $n_{i}$ corresponding to noncrossed diagrams have been taken into account as the retarded and advanced Green's functions in Eq. (14) are calculated self-consistently. In the following, we solve the problem for linear-response theory of a uniform and stationary system in the presence of a uniform electric field.

In the presence of slowly varying perturbations, it is useful to perform the Wigner transformation, viz., the center-ofmass coordinates $[X=(\mathbf{R}, T)]$ and the Fourier transform with respect to the relative coordinates $[k=(\mathbf{k}, \omega)]$. However, the Wigner coordinate $\mathbf{k}$ associated with the momentum operator $-i \boldsymbol{\nabla}$ is not gauge invariant and consequently it is not the correct choice for describing our system. On the other hand, the kinetic momentum $\mathbf{k}(T)=-i \boldsymbol{\nabla}-e \mathbf{A}_{E}(T) /(\hbar c)$ is gauge invariant and, as it will be shown below, for the stationary case all time dependence can be conceived in $\mathbf{k}(T)$; here the vector potential $\mathbf{A}_{E}(T)$ describes the external electric field. The time derivative within the canonical coordinates (marked by wave) becomes a combination of time and momentum derivatives within the kinetic coordinates $\partial_{T}=\partial_{T}+\partial_{T} \mathbf{k}(T) \partial_{\mathbf{k}}$, $\partial_{\tilde{\mathbf{R}}}=\partial_{\mathbf{R}}, \partial_{\mathbf{k}}=\partial_{\mathbf{k}}$, and $\partial_{\widetilde{\omega}}=\partial_{\omega}$.

In the Wigner representation with the kinetic momentum, the convolution of two operators is approximated as

$$
\begin{aligned}
\hat{A} \otimes \hat{B} & =\exp ^{i\left(\partial_{X}^{A} \partial_{k}^{B}-\partial_{k}^{A} \partial_{X}^{B}\right) / 2} \hat{A}(X, k) \hat{B}(X, k) \\
& \approx \hat{A} \hat{B}+\frac{i}{2}\left(\partial_{X} \hat{A} \partial_{k} \hat{B}-\partial_{k} \hat{A} \partial_{X} \hat{B}\right),
\end{aligned}
$$

where we use the four vector notations $\partial_{X} \partial_{k}=\partial_{\mathbf{R}} \partial_{\mathbf{k}}-\partial_{T} \partial_{\omega}$ and $\partial_{T}=\partial_{T}+\frac{e \mathbf{E}}{\hbar} \partial_{\mathbf{k}}$. Here, we assume that a vector potential $\mathbf{A}_{E}(T)=-c \mathbf{E} T$ which corresponds to a uniform electric field E. The first-order gradient expansion is sufficient for the linear-response theory, while the second-order gradient expansion may be necessary for time dependent problems and when the Hamiltonian $\hat{H}_{0}$ is spatially dependent in order to account for the corresponding Berry curvature effects. ${ }^{2}$ Since we are seeking homogeneous solutions both in space and time with respect to the center-of-mass coordinates, the only surviving terms in the expansion are

$$
\hat{A} \otimes \hat{B} \approx \hat{A} \hat{B}-\frac{i}{2 \hbar} e \mathbf{E}\left(\partial_{\mathbf{k}} \hat{A} \partial_{\omega} \hat{B}-\partial_{\omega} \hat{A} \partial_{\mathbf{k}} \hat{B}\right) .
$$

Applying the above Wigner transformation to $\hat{G}_{2}^{<}$in Eq. (13), we obtain directly

$$
\hat{G}_{2}^{<}=n_{F}\left(\hat{G}^{A}-\hat{G}^{R}\right)+\frac{i}{2 \hbar} \partial_{\omega} n_{F} e \mathbf{E}\left(\partial_{\mathbf{k}} \hat{G}_{e q}^{A}+\partial_{\mathbf{k}} \hat{G}_{e q}^{R}\right),
$$

where $\hat{G}_{e q}^{R / A}$ are the Green's functions evaluated at equilibrium, i.e., $\mathbf{E}=0 . \hat{G}_{2}^{<}$solves the Kinetic Eq. (6) up to zeroth order in the electric field $\mathbf{E}$ and therefore the expansion in $\mathbf{E}$ of $\hat{G}_{1}^{<}$and $\hat{\Sigma}_{1}^{<}$starts from the linear in $\mathbf{E}$ terms. With this knowledge, we apply the Wigner transformation to Eq. (14) and find the self-consistent simple form of the kinetic equation for $\hat{G}_{1}^{<}$,

$$
\begin{gathered}
\hat{G}_{1}^{<}=\hat{G}_{e q}^{R} \hat{\Sigma}_{1}^{<} \hat{G}_{e q}^{A}-i e \mathbf{E}\left(\partial_{\omega} n_{F}\right) \hat{G}_{e q}^{R} \hat{\boldsymbol{v}} \hat{G}_{e q}^{A}, \\
\hat{\Sigma}_{1}^{<}=n_{i} \int \frac{d^{2} k^{\prime}}{(2 \pi)^{2}} \hat{T}_{e q}^{R}\left(\mathbf{k}, \mathbf{k}^{\prime}\right) \hat{G}_{1}^{<}\left(\mathbf{k}^{\prime}\right) \hat{T}_{e q}^{A}\left(\mathbf{k}^{\prime}, \mathbf{k}\right),
\end{gathered}
$$

where $\hat{\boldsymbol{v}}=\partial \hat{H}_{0} / \partial \hbar \mathbf{k}$ and $\hat{T}_{e q}^{R / A}$ are self-consistent $T$ matrices evaluated at equilibrium. In the following section, we show how to solve the kinetic Eqs. (19) and (20) for a simple system described by the Rashba Hamiltonian. Whereas solving Eqs. (19) and (20) require only the equilibrium retarded and advance Green's functions and $T$ matrices, note that for $\hat{G}_{2}^{<}$we need to solve these Green's functions up to linear order in $\mathbf{E}$ (see below).

From the equations above, it is natural to decompose the contributions to $\hat{G}^{<}$into the Fermi sea and Fermi-surface contributions $^{1}$ such that $\hat{G}^{<}=\hat{G}_{1}^{<}+\hat{G}_{2}^{<}=\hat{G}_{I}^{<}+\hat{G}_{I I}^{<}$where

$$
\begin{gathered}
\hat{G}_{I}^{<}=\hat{G}_{1}^{<}+\frac{i}{2 \hbar}\left(\partial_{\omega} n_{F}\right) e \mathbf{E}\left(\partial_{\mathbf{k}} \hat{G}_{e q}^{A}+\partial_{\mathbf{k}} \hat{G}_{e q}^{R}\right), \\
\hat{G}_{I I}^{<}=n_{F}\left(\hat{G}^{A}-\hat{G}^{R}\right) .
\end{gathered}
$$

Next, we linearize Eq. (16) in E, carry out the Wigner transformation, and insert the two components of $\hat{G}^{<}$, arriving at the two corresponding components of the current density

$$
\begin{aligned}
& j_{x(y, z)}^{I}=-i e \int \frac{d^{2} \mathbf{k}}{(2 \pi)^{2}} \frac{d \omega}{2 \pi} \operatorname{Tr}\left(\hat{G}_{I}^{<} \hat{v}_{x(y, z)}\right), \\
& j_{x(y, z)}^{I I}=-i e \int \frac{d^{2} \mathbf{k}}{(2 \pi)^{2}} \frac{d \omega}{2 \pi} \operatorname{Tr}\left(\hat{G}_{I I}^{<} \hat{v}_{x(y, z)}\right),
\end{aligned}
$$

where the Fermi surface $\left(j_{x(y, z)}^{I}\right)$ and Fermi sea $\left(j_{x(y, z)}^{I I}\right)$ contributions are identical to ones defined within Kubo-Streda formalism. ${ }^{44}$ Eqs. (19)-(21) are the main results of this subsection.

\section{Linearized Fermi sea contribution}

In order to calculate the Fermi sea contribution using Eqs. (22) and (24), we expand the retarded (advanced) Green's 
function and self-energy up to the first order in $\mathbf{E}$ following the procedure of Onoda et al.: ${ }^{1}$

$$
\begin{aligned}
& \hat{G}^{R, A}=\hat{G}_{e q}^{R, A}+e \mathbf{E} \hat{G}_{\mathbf{E}}^{R, A}+O\left(E^{2}\right), \\
& \hat{\Sigma}^{R, A}=\hat{\Sigma}_{e q}^{R, A}+e \mathbf{E} \hat{\Sigma}_{\mathbf{E}}^{R, A}+O\left(E^{2}\right),
\end{aligned}
$$

where $\quad \hat{G}_{\mathbf{E}}^{R, A}=\left.\frac{1}{e} \partial_{\mathbf{E}} \hat{G}^{R, A}\right|_{E=0}, \quad \hat{\Sigma}_{\mathbf{E}}^{R, A}=\left.\frac{1}{e} \partial_{\mathbf{E}} \hat{\Sigma}^{R, A}\right|_{E=0}, \quad$ and $\hat{G}_{e q}^{R, A}\left(\hat{\Sigma}_{e q}^{R, A}\right)$ are the Green's functions (self-energies) evaluated at equilibrium, i.e., $\mathbf{E}=0$. The Fermi sea lesser Green's function $\hat{G}_{I I}^{<}$calculated up to the first order in the electric field $\mathbf{E}$ becomes

$$
\hat{G}_{I I}^{<}=n_{F}\left(\hat{G}_{e q}^{A}-\hat{G}_{e q}^{R}\right)+n_{F} e \mathbf{E}\left(\hat{G}_{\mathbf{E}}^{A}-\hat{G}_{\mathbf{E}}^{R}\right) .
$$

We now substitute Eq. (25) into Eqs. (5) and (11) only retaining linear terms in $\mathbf{E}$ in order to arrive at the following self-consistent equations:

$$
\begin{aligned}
\hat{G}_{\mathbf{E}}^{R, A}= & \hat{G}_{e q}^{R, A} \hat{\Sigma}_{\mathbf{E}} \hat{G}_{e q}^{R, A}-\frac{i}{2}\left[\hat{G}_{e q}^{R, A}\left(\hat{v}+\partial_{\hbar k} \hat{\Sigma}_{e q}^{R, A}\right) \partial_{\omega} \hat{G}_{e q}^{R, A}\right. \\
& \left.-\partial_{\omega} \hat{G}_{e q}^{R, A}\left(\hat{v}+\partial_{\hbar k} \hat{\Sigma}_{e q}^{R, A}\right) \hat{G}_{e q}^{R, A}\right], \\
\hat{\Sigma}_{\mathbf{E}}^{R, A}= & n_{i} \int \frac{d^{2} k^{\prime}}{(2 \pi)^{2}} \hat{T}_{e q}^{R, A}\left(\mathbf{k}, \mathbf{k}^{\prime}\right) \hat{G}_{\mathbf{E}}^{R, A}\left(\mathbf{k}^{\prime}\right) \hat{T}_{e q}^{R, A}\left(\mathbf{k}^{\prime}, \mathbf{k}\right),
\end{aligned}
$$

where in Eq. (5) we also performed the gradient expansion and linearized form of Eq. (11) was substituted in $\left\langle\mathbf{k}\left|\hat{\Sigma}^{R, A}\right| \mathbf{k}^{\prime}\right\rangle=n_{i}\left\langle\mathbf{k}\left|\hat{T}^{R, A}\right| \mathbf{k}\right\rangle \delta\left(\mathbf{k}-\mathbf{k}^{\prime}\right)$. Equations (26)-(28) are the main results of this subsection.

\section{AHE IN RASHBA SYSTEMS}

In this section, we apply the above formalism to $2 \mathrm{DEG}$ with exchange field and spin-orbit interaction. A general numerical procedure is followed by analytical results valid in the metallic regime in the limit of small impurity scattering broadening $\hbar / \tau$ with respect to the Fermi energy $\varepsilon_{F}$. We end the section with a discussion of the numerical and analytical results comparing them to other approaches. For convenience and in order to keep the expressions more concise, we introduce here the dimensionless units that can easily be transformed into dimensional units by following equations at the beginning of this section. Note that our formalism cannot be used close to the energies $\omega= \pm h$ in Fig. 2, as $k_{F} l$ ( $l$ is the mean-free path) can become very small and the noncrossing approximation in Fig. 1 may fail. Nevertheless, we do not expect large corrections to our results around these singularities as the nondiagonal conductivity seems not to be strongly affected by including the crossed diagrams. ${ }^{45}$

\section{A. Calculational procedure}

We restrict ourselves here to 2DEG Rashba Hamiltonian with an exchange field $\breve{h}$ (breve accent here means that $h$ is in dimensional units) in order to obtain simple analytical results that connect directly with other microscopic linearresponse calculations $19,22,46$

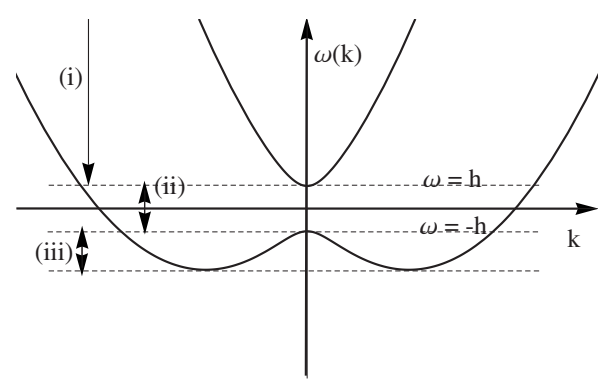

FIG. 2. Electronic band dispersions of the Rashba model; throughout the paper, $\varepsilon_{F}$ is the Fermi energy measured from the bottom of the lower band while $\omega_{F}$ is the Fermi energy measured from the middle of the gap [region (ii)].

$$
\hat{H}_{R}=\hat{1}(\hbar \breve{\mathbf{k}})^{2} / 2 m+\breve{\alpha} \breve{\mathbf{k}} \cdot \hat{\boldsymbol{\sigma}} \times \mathbf{z}-\breve{h} \hat{\sigma}_{z}+\hat{1} V(\breve{\mathbf{r}}),
$$

where $\breve{\alpha}$ is the strength of spin-orbit interaction, $\hat{\boldsymbol{\sigma}}$ are Pauli matrices, $\hbar \breve{\mathbf{k}}=-i \hbar \nabla-e \mathbf{A} / c, \mathbf{A}(t)=-c \mathbf{E} t$ describes the external electric field, and $V(\mathbf{r})$ describes the impurities. From symmetry considerations, the most general form of the Hamiltonian in Eq. (29) should treat the coordinate $\mathbf{r}$ as an operator $\mathbf{r}+\hat{\mathbf{r}}_{s o}(k)$, with $\hat{\mathbf{r}}_{s o}(\mathbf{k})=\lambda \hat{\boldsymbol{\sigma}} \times \mathbf{k}$ originating from the projection procedure onto the band under consideration. ${ }^{47}$ The spin-orbit interaction can also include higher, e.g., cubic terms relevant for the bulk $\mathrm{InSb}$ and the $\mathrm{HgTe}$ quantum wells with an inverted band structure. ${ }^{48,49}$ Here, only linear terms with Rashba symmetry are considered with $\hat{\mathbf{r}}_{s o}(\mathbf{k})$ being disregarded as we expect effect of $H_{s o}=\hat{\mathbf{r}}_{s o}(\mathbf{k}) \boldsymbol{\nabla} V(\mathbf{r})$ on the AHE to be small for wide band semiconductors in which $\lambda$ is relatively small. ${ }^{50}$ The disorder in the system is modeled by impurity delta scatterers

$$
V(\mathbf{r})=\breve{V}_{0} \sum_{i} \delta\left(\breve{\mathbf{r}}-\breve{\mathbf{r}}_{i}\right)
$$

where $\breve{\mathbf{r}}_{i}$ describes the positions of randomly distributed impurities of density $\breve{n}_{i}$.

We rewrite the Hamiltonian in dimensionless quantities

$$
\frac{\hat{H}_{R}}{\varepsilon_{F}}=\hat{1} \frac{1}{2} \mathbf{k}^{2}+\alpha \mathbf{k} \cdot \hat{\boldsymbol{\sigma}} \times \mathbf{z}-h \hat{\boldsymbol{\sigma}}_{z}+\hat{1} V_{0} \sum_{i} \delta\left(\mathbf{r}-\mathbf{r}_{i}\right),
$$

where $\varepsilon_{F}$ is the Fermi energy measured from the minimum of energy and $k=\breve{k} l_{0}$ is the dimensionless momentum. The dimensionality can be restored by substituting expressions for the dimensionless units into the final formulas

$$
\begin{gathered}
l_{0}=\sqrt{\frac{\hbar^{2}}{m \varepsilon_{F}}}, \quad \alpha=\breve{\alpha} \sqrt{\frac{m}{\hbar^{2} \varepsilon_{F}}}, \quad V_{0}=\frac{m \breve{V}_{0}}{\hbar^{2}}, \\
h=\frac{\breve{h}}{\varepsilon_{F}}, \quad n_{i}=\breve{n}_{i} l_{0}^{2}, \quad k=\breve{k} l_{0} .
\end{gathered}
$$

Also note that whereas $\varepsilon_{F}$ is measured from the bottom of the lower band, in the notation below, we introduce $\omega_{F}$ which is the Fermi energy measured from the middle of the gap [region (ii) in Fig. 2]. In the following, we solve Eqs. (19) and (20) in order to find the nonequilibrium Green's 
function $\hat{G}_{1}^{<}$describing processes at the Fermi surface and Eqs. (27) and (28) for the nonequilibrium Green's function $\hat{G}_{2}^{<}$-primarily Fermi sea contribution.

We calculate $\hat{\Sigma}_{e q}^{R, A}$ and the Green's functions $\hat{G}_{e q}^{R, A}$ using the self-consistent TMA, i.e., diagonal components of Eq. (8) (Refs. 1 and 20)

$$
\begin{gathered}
\hat{T}_{e q}^{R, A}=V_{0}\left(\hat{1}-V_{0} \hat{\gamma}^{R, A}\right)^{-1}, \\
\hat{\Sigma}_{e q}^{R, A}(\omega)=n_{i} \hat{T}_{e q}^{R, A}(\omega)=\Sigma_{e q 0}^{R, A} \hat{\sigma}_{0}+\Sigma_{e q z}^{R, A} \hat{\sigma}_{z}, \\
\hat{G}_{e q}^{R}=\left(\omega \hat{1}-\hat{H}_{0}-\hat{\Sigma}_{e q}^{R}\right)^{-1} \\
=\frac{\left(W-\frac{k^{2}}{2}\right) \hat{\sigma}_{0}+\alpha k_{y} \hat{\sigma}_{x}-\alpha k_{x} \hat{\sigma}_{y}-H \hat{\sigma}_{z}}{\left(W-\frac{k^{2}}{2}\right)^{2}-H^{2}-\alpha^{2} k^{2}}, \\
\hat{G}_{e q}^{A}=\left(\hat{G}_{e q}^{R}\right)^{\dagger},
\end{gathered}
$$

where $\quad W=\omega-\Sigma_{e q 0}^{R}, \quad H=h-\Sigma_{e q \mathrm{Z}}^{R}, \quad$ and $\quad \hat{\gamma}^{R, A}=\int d^{2} k /$ $(2 \pi)^{2} \hat{G}_{e q}^{R, A}(\mathbf{k}, \omega) \equiv \gamma^{R, A} \hat{\sigma}_{0}+\gamma_{z}^{R, A} \hat{\sigma}_{z}$. We calculate selfconsistent value of the self-energy $\hat{\Sigma}_{e q}^{R, A}(\omega)$ for each $\omega$ by performing sufficient number of iterations in Eq. (33) in order to achieve the prescribed accuracy (see Appendix B for details).

With the knowledge of the equilibrium Green's function $\hat{G}_{e q}^{R}(\mathbf{k}, \omega)$, we can calculate the local densities of states

$$
D(\omega) \equiv-\frac{1}{\pi} \int \frac{d^{2} k}{(2 \pi)^{2}} \operatorname{Im}\left\{\operatorname{Tr}\left[\hat{G}_{e q}^{R}(\mathbf{k}, \omega)\right]\right\}
$$

and the total number of electrons

$$
N=\int_{-\infty}^{\omega_{F}} d \omega D(\omega) .
$$

The number of electrons changes as we increase the disorder and following Eq. (35), $\omega_{F}$ is always adjusted so that the total number of electrons is constant.

The same TMA is also used to calculate $\hat{\Sigma}^{<}$and $\hat{\Sigma}_{\mathbf{E}}^{R, A}$ from Eqs. (20) and (28), respectively,

$$
\begin{gathered}
\hat{\Sigma}_{1}^{<}=n_{i} \int \frac{d^{2} k}{(2 \pi)^{2}} \hat{T}_{e q}^{R}(\omega) \hat{G}_{1}^{<}(\mathbf{k}, \omega) \hat{T}_{e q}^{A}(\omega), \\
\hat{\Sigma}_{\mathbf{E}}^{R, A}=n_{i} \int \frac{d^{2} k}{(2 \pi)^{2}} \hat{T}_{e q}^{R, A}(\omega) \hat{G}_{\mathbf{E}}^{R, A}(\mathbf{k}, \omega) \hat{T}_{e q}^{R, A}(\omega) .
\end{gathered}
$$

The TMA with self-consistent calculation of the equilibrium Green's functions $\hat{G}_{e q}^{R, A}$ described in Appendix B allows us to take into account higher order noncrossed diagrams in the concentration of impurities $n_{i}$, with weak localization diagrams being disregarded. The procedure of calculating the retarded (advanced) and nonequilibrium self-energies in Eqs. (33) and (36) is represented graphically in Fig. 3. In this graphical representation, the bold arrow corresponds to the

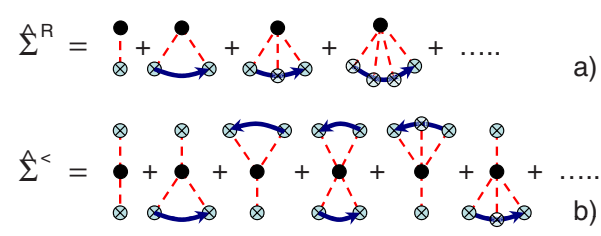

FIG. 3. (Color online) An infinite set of diagrams representing the self-consistent TMA in calculating (a) the retarded (advanced) self-energy $\hat{\Sigma}_{e q}^{R, A}$ and (b) the lesser component of self-energy $\hat{\Sigma}^{<}$in Eqs. (33) and (36), respectively.

self-consistently calculated retarded (advanced) Green's function.

For the delta scatterers, $T$ matrix does not depend on momentum $\mathbf{k}$ which allows us to perform momentum integrations in Eqs. (36) and (37). It is then useful to introduce the following $2 \times 2$ matrices:

$$
\begin{gathered}
\hat{\rho}(\omega) \equiv \int \frac{d^{2} k}{(2 \pi)^{2}} \hat{G}_{1}^{<}(\mathbf{k}, \omega), \\
\hat{\rho}_{\mathbf{E}}^{R, A}(\omega) \equiv \int \frac{d^{2} k}{(2 \pi)^{2}} \hat{G}_{\mathbf{E}}^{R, A}(\mathbf{k}, \omega) .
\end{gathered}
$$

The elements of matrices $\hat{\rho}$ and $\hat{\rho}_{\mathrm{E}}$ satisfy a system of linear equations obtained by integrating in momentum space the left- and right-hand sides of Eqs. (19) and (27), respectively,

$$
\begin{aligned}
\hat{\rho}= & \int \frac{d^{2} k}{(2 \pi)^{2}} \hat{G}_{e q}^{R} \hat{T}_{e q}^{R}(\omega) \hat{\rho}(\omega) \hat{T}_{e q}^{A}(\omega) \hat{G}_{e q}^{A} \\
& -i \partial_{\omega} n_{F} e \mathbf{E} \int \frac{d^{2} k}{(2 \pi)^{2}} \hat{G}_{e q}^{R} \hat{\boldsymbol{v}} \hat{G}_{e q}^{A},
\end{aligned}
$$

$$
\begin{aligned}
\hat{\rho}_{\mathbf{E}}^{R, A}= & \int \frac{d^{2} k}{(2 \pi)^{2}} \hat{G}_{e q}^{R, A} \hat{T}_{e q}^{R, A}(\omega) \hat{\rho}_{\mathbf{E}}^{R, A}(\omega) \hat{T}_{e q}^{R, A}(\omega) \hat{G}_{e q}^{R, A} \\
& -\frac{i}{2} \int \frac{d^{2} k}{(2 \pi)^{2}}\left(\hat{G}_{e q}^{R, A} \hat{\boldsymbol{v}} \partial_{\omega} \hat{G}_{e q}^{R, A}-\partial_{\omega} \hat{G}_{e q}^{R, A} \hat{\boldsymbol{v}} \hat{G}_{e q}^{R, A}\right) .
\end{aligned}
$$

The momentum integrations in the right-hand side of Eqs. (40) and (41) are done analytically using the general form of the Green's functions $\hat{G}_{e q}^{R, A}(\mathbf{k}, \omega)$ in Eq. (34). Without loss of generality, we take the electric field $\mathbf{E}$ along the $y$ axis $\mathbf{E}$ $=\left(0, E_{y}\right)$ and solve the system of linear Eqs. (40) and (41) for the elements of matrices $\hat{\rho}$ and $\hat{\rho}_{\mathbf{E}}$ in Appendixes $\mathrm{C}$ and $\mathrm{D}$, respectively.

With this, we calculate the current from Eqs. (23) and (24), respectively, with a use of Eqs. (19), (21), (26), and (27),

$$
\begin{aligned}
j_{x(y)}^{I}= & -i e \int \frac{d^{2} k}{(2 \pi)^{2}} \frac{d \omega}{2 \pi} \operatorname{Tr}\left\{\hat{G}_{e q}^{R} \hat{T}_{e q}^{R} \hat{\rho} \hat{T}_{e q}^{A} \hat{G}_{e q}^{A} \hat{\boldsymbol{v}}_{x(y)}\right. \\
& \left.-i e \mathbf{E} \partial_{\omega} n_{F}\left[\hat{G}_{e q}^{R} \hat{\boldsymbol{v}} \hat{G}_{e q}^{A}-\frac{1}{2}\left(\hat{G}_{e q}^{A} \hat{\boldsymbol{v}} \hat{G}_{e q}^{A}+\hat{G}_{e q}^{R} \hat{\boldsymbol{v}} \hat{G}_{e q}^{R}\right)\right] \hat{\boldsymbol{v}}_{x(y)}\right\},
\end{aligned}
$$




$$
\begin{aligned}
j_{x(y)}^{I I}= & i e \int \frac{d^{2} k}{(2 \pi)^{2}} \frac{d \omega}{2 \pi} e \mathbf{E} n_{F} \operatorname{Tr}\left[\hat{G}_{e q}^{R} \hat{T}_{e q}^{R} \hat{\rho}_{\mathbf{E}}^{R} \hat{T}_{e q}^{R} \hat{G}_{e q}^{R} \hat{\boldsymbol{v}}_{x(y)}\right. \\
& \left.-\frac{i}{2}\left(\hat{G}_{e q}^{R} \hat{\boldsymbol{v}} \partial_{\omega} \hat{G}_{e q}^{R}-\partial_{\omega} \hat{G}_{e q}^{R} \hat{\boldsymbol{v}} \hat{G}_{e q}^{R}\right) \hat{v}_{x(y)}\right]+ \text { c.c. },
\end{aligned}
$$

where we use $\partial_{\mathbf{k}} \hat{G}_{e q}^{R, A}=\hat{G}_{e q}^{R, A} \hat{\boldsymbol{v}} \hat{G}_{e q}^{R, A}$, which holds for the model of delta impurities. In Eq. (42), we perform analytical integrations over momentum $\mathbf{k}$ and energy $\omega$ while in Eq. (43), we only perform analytical integration over momentum. The results of these integrations are given in Appendixes $\mathrm{E}$ and $\mathrm{F}$ for Eqs. (42) and (43), respectively.

\section{B. Analytical results in the metallic regime}

In the metallic regime, we are able to obtain analytical results as it is sufficient to consider only finite number of terms in the expansion with respect to the strength of impurity in Fig. 3. For the same reason, we are also able to generalize the disorder in Eq. (30) (generalization of the theory is given in Appendix A) as follows:

$$
V(\mathbf{r})=\sum_{i} V_{0}^{i} \delta\left(\mathbf{r}-\mathbf{r}_{i}\right)
$$

where $\mathbf{r}_{i}$ is random, the strength of each impurity has the same arbitrary distribution, and all strength distributions are independent leading to the first four cumulants $\left\langle V_{0}^{i}\right\rangle_{d i s}=0$, $n_{i}\left\langle\left(V_{0}^{i}\right)^{2}\right\rangle_{d i s}=V_{2}, n_{i}\left\langle\left(V_{0}^{i}\right)^{3}\right\rangle_{d i s}=V_{3}$, and $n_{i}\left\langle\left(V_{0}^{i}\right)^{4}\right\rangle_{d i s}=V_{4}$, where $n_{i}$ is the concentration of impurities. For the disorder described in Eq. (30), we have $\sqrt{V_{2} / n_{i}}=\sqrt[3]{V_{3} / n_{i}}=\sqrt[4]{V_{4} / n_{i}}=V_{0}$ and for the telegraph white-noise disorder we have $V_{3}=0$ as it is mentioned in Appendix A.

In this section, we first expand the retarded (advanced) self-energy in Eq. (33) up to the third order in $V_{0}$ [or up to the terms $V_{3}$ in Eq. (A4)]. The lesser component of the selfenergy in Eq. (36) has to be expanded up to the fourth order in $V_{0}$ [or up to the terms $V_{4}$ in Eq. (A5)], which corresponds to the four legged diagrams in Fig. 3(b). This ensures that the expansion of the conductivity $\sigma_{x y}^{I}$ following from Eqs. (E3) and (E5) captures all possible terms proportional to $1 / V_{0}$ and 1.

The expansion of $\sigma_{x y}^{I I}$ following from Eq. (F3) is somewhat simpler as it only contains the terms proportional to 1 and its calculation requires consideration of only one bare bubble diagram (e.g., summation of vertices leads to higher order corrections). In our discussion, we thus concentrate on the diagrams for calculating $\sigma_{x y}^{I}$ and also present the result for the bare bubble diagram of $\sigma_{x y}^{I I}$. Note that in the expansion of $\sigma^{I(I I)}$, it is important to properly consider the branch cut of the "In" function taken as $(-\infty, 0]$. The diagrams in Fig. 3 have direct correspondence to the Kubo formalism diagrams in Fig. 4 used in Ref. 22. This allows us to separate the conductivity into terms that directly relate to each diagram in Fig. 4.

We distinguish three regimes for the position of the Fermi energy with respect to the gap of the size $2 h$ : (i) $\omega_{F}>h$, (ii) $-h<\omega_{F}<h$, and (iii) $\omega_{F}<-h$ (see Fig. 2). To simplify formulas, we introduce the following notation:

$$
k_{ \pm}^{2}=2\left(\omega_{F}+\alpha^{2} \mp \sqrt{h^{2}+2 \omega_{F} \alpha^{2}+\alpha^{4}}\right),
$$

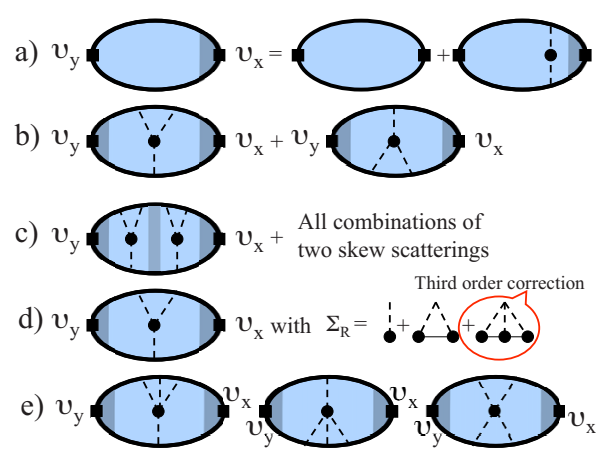

FIG. 4. (Color online) Different diagrammatic contributions to $\sigma_{x y}^{I}$ within the Kubo formula formalism: (a) the ladder diagram (vertex) contribution $\sim 1$, (b) the skew-scattering contribution $\sim 1 /\left(n_{i} V_{0}\right) \sim V_{3} / V_{2}^{2}$, (c) the double skew-scattering contribution $\sim 1 / n_{i} \sim V_{3}^{2} / V_{2}^{3}$, (d) the skew-scattering contribution in which the retarded (advanced) self-energy is calculated up to the third order $\sim 1 / n_{i} \sim V_{3}^{2} / V_{2}^{3}$, and (e) the fourth-order skew-scattering contribution $\sim 1 / n_{i} \sim V_{4} / V_{2}^{2}$.

$$
\begin{gathered}
\lambda_{ \pm}=\sqrt{\left(\alpha k_{ \pm}\right)^{2}+h^{2}}, \quad \lambda_{F}=\sqrt{2 \omega_{F} \alpha^{2}+h^{2}}, \\
\kappa_{ \pm}=\sqrt{\left(\alpha k_{ \pm}\right)^{2}+4 h^{2}}, \\
\nu_{ \pm}=k\left|\frac{d \omega(k)}{d k}\right|^{-1}=\left\{\begin{array}{l}
\frac{\lambda_{ \pm}}{\lambda_{ \pm} \pm \alpha^{2}}, \quad \omega_{F}>h \\
\frac{\lambda_{-}}{\lambda_{-}-\alpha^{2}},-h<\omega_{F}<h \\
\frac{\lambda_{ \pm}}{\left|\lambda_{ \pm}-\alpha^{2}\right|}, \quad \omega_{F}<-h,
\end{array}\right.
\end{gathered}
$$

where $\nu_{ \pm}$is the density of states at the Fermi level and $k_{ \pm}$ are the two Fermi wave numbers for regimes (i) and (iii). In regime (ii), $k_{+}$becomes pure imaginary and only $k_{-}$has the meaning of the Fermi wave number. Further, we introduce the following parameter:

$$
\Lambda=\frac{V_{3}}{V_{2}^{2}} \gamma_{z}^{j}+\frac{V_{4}}{V_{2}^{2}}\left(3 \gamma^{r} \gamma_{z}^{i}+\gamma^{j} \gamma_{z}^{r}\right)
$$

where $\hat{\gamma}=\int d^{2} k /(2 \pi)^{2} \hat{G}_{e q}^{R} \equiv \gamma \hat{\sigma}_{0}+\gamma_{z} \hat{\sigma}_{z}$, with $\gamma=\gamma^{r}+i \gamma^{i}$ and $\gamma_{z}=\gamma_{z}^{r}+i \gamma_{z}^{i}$. Note that the two-dimensional integral over momentum diverges and $\hat{\gamma}$ is calculated by introducing the momentum cutoff (see Appendix B). By expanding the result of Appendix B up to the zeroth order in the strength of impurities, we obtain

$$
\gamma=\frac{\left(k_{-}^{2}-2 \omega_{F}\right) \ln \left|\frac{k_{-}^{2}}{k_{0}^{2}-k_{-}^{2}}\right|-\left(k_{+}^{2}-2 \omega_{F}\right) \ln \left|\frac{k_{+}^{2}}{k_{0}^{2}-k_{+}^{2}}\right|}{2 \pi\left(k_{-}^{2}-k_{+}^{2}\right)},
$$

$$
\gamma_{z}^{r}=\frac{h}{\pi\left(k_{+}^{2}-k_{-}^{2}\right)} \ln \left|\frac{k_{+}^{2}\left(k_{0}^{2}-k_{-}^{2}\right)}{k_{-}^{2}\left(k_{0}^{2}-k_{+}^{2}\right)}\right|,
$$




$$
\begin{aligned}
& \dot{\gamma}=\left\{\begin{array}{c}
-\frac{\nu_{-}+\nu_{+}}{4}, \omega_{F}>h \\
-\frac{\nu_{-}}{4},-h<\omega_{F}<h \\
-\frac{k_{-}^{2}+k_{+}^{2}-4 \omega_{F}}{2\left(k_{-}^{2}-k_{+}^{2}\right)}, \omega_{F}<-h,
\end{array}\right. \\
& \gamma_{z}^{i}=\left\{\begin{array}{l}
\frac{h}{4}\left(\frac{\nu_{+}}{\lambda_{+}}-\frac{\nu_{-}}{\lambda_{-}}\right), \quad \omega_{F}>h \\
-\frac{h}{4} \frac{\nu_{-}}{\lambda_{-}}, \quad-h<\omega_{F}<h \\
-\frac{2 h}{k_{-}^{2}-k_{+}^{2}}, \quad \omega_{F}<-h,
\end{array}\right.
\end{aligned}
$$

where $k_{0}$ is the cutoff in the momentum integration.

As it follows from the Appendixes $\mathrm{E}$ and $\mathrm{F}$, the nondiagonal conductivities $\sigma_{x y}^{I(I I)}$ can be calculated by properly choosing the "ln" branch that corresponds to the regimes (i), (ii), or (iii), respectively. The result of expanding Eqs. (E3) and (E5), and Eq. (F3) for conductivities $\sigma_{x y}^{I}$ and $\sigma_{x y}^{I I}$, respectively, in the region (i) $\left(\omega_{F}>h\right)$ becomes

$$
\begin{gathered}
\sigma_{x y}^{I(i)}=\frac{2 e^{2} \alpha^{2}}{\hbar \pi} \Lambda=-\frac{V_{4} e^{2} h \alpha^{2} \ln \left|\frac{k_{+}^{2}\left(k_{0}^{2}-k_{-}^{2}\right)}{k_{-}^{2}\left(k_{0}^{2}-k_{+}^{2}\right)}\right|}{\hbar \pi^{2}\left(k_{+}^{2}-k_{-}^{2}\right)}, \\
\sigma_{x y}^{I I(i)}=0,
\end{gathered}
$$

which reproduces result of Ref. 24 in the limit of large cutoff $k_{0}$. In reference to the Kubo formula formalism, we can claim the following: the diagrams in Fig. 4(a) vanish after summation (the intrinsic and side-jump contributions defined in Ref. 46 cancel each other), ${ }^{24}$ the diagrams in Figs. 4(b)-4(d) are all proportional to $\frac{\nu_{+}}{\lambda_{+}}-\frac{\nu_{-}}{\lambda_{-}} \equiv 0$ and also vanish, and the diagrams in Fig. 4(e) lead to the result in Eq. (45). $\sigma_{x y}^{I I(i)}$ is zero as the corresponding bare bubble contribution in Eq. (F3) vanishes. Repeating the same procedure for the region (ii) $\left(-h<\omega_{F}<h\right)$, we obtain

$$
\begin{aligned}
\sigma_{x y}^{I(i i)}= & \frac{e^{2}}{4 \pi \hbar}\left(\frac{h \alpha^{2} \nu_{-}}{\lambda_{-}^{2}}-\frac{4 h k_{-}^{2} \alpha^{2}}{\lambda_{-} \kappa_{-}^{2}}+\frac{3 h k_{-}^{4} \alpha^{2}}{\kappa_{-}^{4} \nu_{-}}+\frac{8 k_{-}^{4} \alpha^{2} \lambda_{-}^{2}}{\kappa_{-}^{4} \nu_{-}^{2}} \Lambda\right. \\
& +\left[\frac{8 h\left(2 h^{2}+2 \omega_{F} \alpha^{2}+k_{-}^{2} \alpha^{2}\right)}{\kappa_{-}^{2}} \gamma_{z}^{j}+\left(k_{-}^{2}-k_{+}^{2}\right) \gamma^{j}\right] \\
& \left.\times \frac{2 h k_{-}^{4} \alpha^{2}}{\kappa_{-}^{4}} \frac{V_{3}^{2}}{V_{2}^{3}}\right), \\
\sigma_{x y}^{I I(i i)}= & \frac{e^{2}}{4 \pi \hbar}\left(1-\frac{h}{\sqrt{\alpha^{4}+\lambda_{F}^{2}}}\right),
\end{aligned}
$$

where the diagrams in Fig. 4(a) lead to the first three disorder independent terms in Eq. (46) (the intrinsic, the side-jump, and the disorder independent skew-scattering terms, respectively), ${ }^{24}$ the skew-scattering diagrams in Figs. 4(b) and 4(e) lead to the term in Eq. (46) proportional to $\Lambda$ and the diagrams in Figs. 4(c) and 4(d) lead to the terms in Eq. (46) proportional to $V_{3}^{2} / V_{2}^{3} \cdot \sigma_{x y}^{I I(i i)}$ is calculated from a bare bubble contribution given by Eq. (F3) and also corresponds to the intrinsic contribution. Finally for the region (iii) $\left(\omega_{F}\right.$ $<-h$ ), we obtain

$$
\begin{aligned}
\sigma_{x y}^{I(i i i)}= & \frac{e^{2}}{4 \pi \hbar}\left(\frac{32 h \omega_{F}^{2} \alpha^{4}}{\left(h^{2}+\alpha^{4}\right)^{2}\left(k_{-}^{2}-k_{+}^{2}\right)}+\frac{\alpha^{2}\left(k_{-}^{2}-k_{+}^{2}\right)^{4}}{32\left(h^{2}+\alpha^{4}\right)^{2}} \Lambda\right. \\
+ & {\left[\frac{h\left(h^{2} \omega_{F}+2 \alpha^{2} h^{2}-3 \omega_{F} \alpha^{4}\right)}{\left(h^{2}-\omega_{F} \alpha^{2}\right)\left(h^{2}+\alpha^{4}\right)} \gamma_{z}^{j}+\gamma^{j}\right] } \\
& \times \frac{h \alpha^{2}\left(k_{-}^{2}-k_{+}^{2}\right)^{3}}{4\left(h^{2}+\alpha^{4}\right)^{2}} \frac{V_{3}^{2}}{V_{2}^{3}}, \\
& \sigma_{x y}^{I I(i i i)}=\frac{e^{2}}{4 \pi \hbar} \frac{h\left(\lambda_{-}-\lambda_{+}\right)}{\left(\alpha^{2}-\lambda_{-}\right)\left(\alpha^{2}-\lambda_{+}\right)},
\end{aligned}
$$

where the diagrams in Fig. 4(a) lead to the disorder independent term in Eq. (47) (it includes the intrinsic, the side-jump, and the disorder independent skew-scattering contributions), the skew-scattering diagrams in Figs. 4(b) and 4(e) lead to the term in Eq. (47) proportional to $\Lambda$, and the diagrams in Figs. 4(c) and 4(d) lead to the terms in Eq. (47) proportional to $V_{3}^{2} / V_{2}^{3} . \sigma_{x y}^{I I(i i)}$ is again calculated from a bare bubble contribution given by Eq. (F3).

The diagonal conductivities can also be calculated by expanding Eqs. (E4) and (E6),

$$
\sigma_{y y}=\left\{\begin{array}{c}
\frac{e^{2}}{\hbar} \frac{\omega_{F}+\alpha^{2}}{\pi V_{2}}, \omega_{F}>h \\
\frac{e^{2}}{\hbar} \frac{k_{-}^{2} \lambda_{-}^{2}}{\pi V_{2} \nu_{-}^{2} \kappa_{-}^{2}}, \quad-h<\omega_{F}<h \\
\frac{e^{2}}{\hbar} \frac{\left(\omega_{F}+\alpha^{2}\right)\left(\alpha^{4}+\lambda_{F}^{2}\right)}{\pi V_{2}\left(\alpha^{4}+h^{2}\right)}, \omega_{F}<-h,
\end{array}\right.
$$

where we only present the dominant nonvanishing terms $V_{2}^{-1}$ as the higher order terms are quite cumbersome.

\section{Numerical results and discussions}

Here, we present results of our numerical calculations based on the formalism developed in Sec. III A. Figures 5-8 show the numerical results for the anomalous Hall conductivity as a function of the Fermi energy $\omega_{F}$ and the first Born scattering amplitude $\gamma_{\mathrm{Born}}=n_{i} V_{0}^{2}$. The strength of the spinorbit interaction is chosen to be the same as in Ref. 1, $2 \alpha^{2} / h=35.9\left(2 \alpha^{2} / E_{\mathrm{res}}=3.59, E_{\mathrm{res}}=10 h\right)$ and the strengths of impurity are $V_{0}=0.1,0.3,-0.1$, and -0.3 . For the retarded (advanced) self-energy, the cutoff in the momentum integration is $k_{0}=12$ which corresponds to the energy cutoff of Ref. 1, $\varepsilon_{c}=3 E_{\text {res }}$. The Born scattering amplitude is varied by changing the impurity concentration $n_{i}$.

In the clean limit, when $\gamma_{\text {Born }} \rightarrow 0$, we observe skewscattering behavior $\left[\sigma_{x y} \sim 1 /\left(n_{i} V_{0}\right)\right]$ in which $\left|\sigma_{x y}\right|$ rapidly increases. For repulsive scatterers $\left(V_{0}>0\right.$, see Figs. 5 and 6), the negative conductivity diminishes as we increase the Fermi energy until the point $\omega_{F}=-h$ is reached. At this point, the conductivity suddenly increases without a change of sign, 


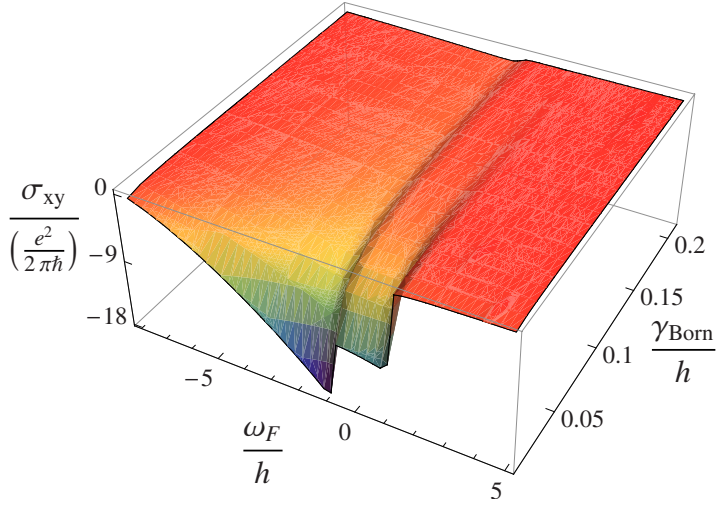

FIG. 5. (Color online) The anomalous Hall conductivity $\sigma_{x y}$ as a function of the Fermi energy $\omega_{F}$ and the Born scattering amplitude $\gamma_{\text {Born. }}$ The parameters are chosen as $2 \alpha^{2} / h=35.9, k_{0}=12$, and $V_{0}$ $=0.1$. The Fermi energy $\omega_{F}$ corresponds here to the clean system and it is renormalized according to Eq. (35) in the presence of disorder.

in contrast to Ref. 1 where the sign change has been observed but in agreement with Refs. 22 and 24 [note that Fig. 5 is calculated for exactly the same parameters as Fig. 5(c) in Ref. 1]. As we increase the Fermi energy further, the conductivity increases again around $\omega_{F}=h$ acquiring a very small negative value. In this regime, both subbands are partially occupied and only the higher order skew scattering ${ }^{22,24}$ (hybrid skew scattering) contributes to the anomalous Hall effect. Relatively large hybrid skew scattering is present in Fig. 6 compared to Fig. 5 as the hybrid skew-scattering contribution is proportional to $1 / n_{i} \sim V_{0}^{2} / \gamma_{\text {Born }}$ and should be larger for greater impurity strength. ${ }^{24}$ The same is true for the conventional skew scattering proportional to $1 /\left(V_{0} n_{i}\right)$ $\sim V_{0} / \gamma_{\text {Born }}$, which can be immediately seen from Figs. 5-8. For attractive scatterers $\left(V_{0}<0\right.$, see Figs. 7 and 8$)$ the sign of the ordinary skew scattering dominating in the clean limit is opposite to the sign of the ordinary skew scattering for the repulsive scatterers. The conductivity now increases until we reach the point $\omega_{F}=-h$ in which we observe a sudden drop. One more drop happens around the point $\omega_{F}=h$ where the anomalous Hall conductivity changes sign (see Figs. 7 and 8). This change of sign is consistent with the fact that the higher order (hybrid) skew scattering (prevailing when both

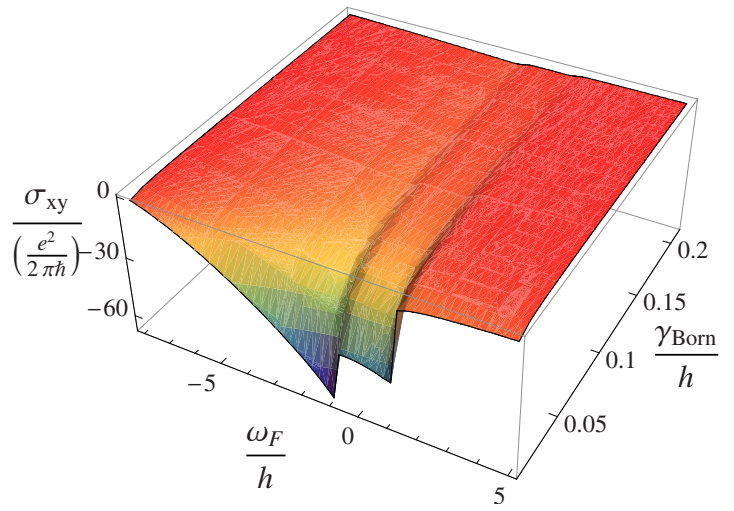

FIG. 6. (Color online) Identical to Fig. 5 plot but for larger strength of impurity $V_{0}=0.3$.

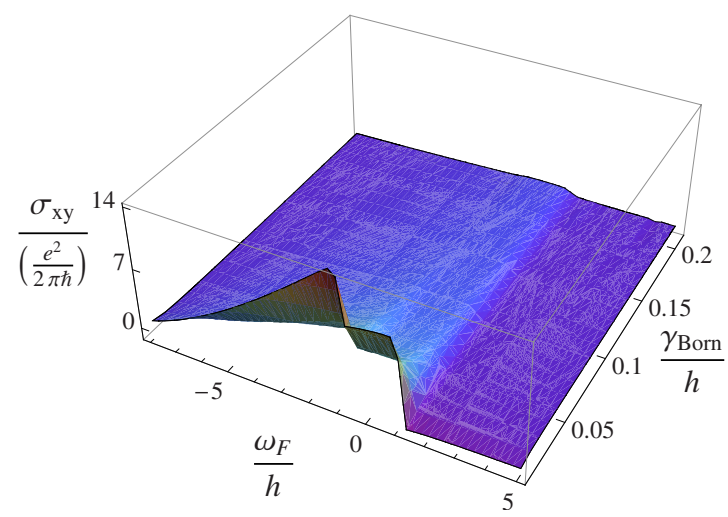

FIG. 7. (Color online) Identical to Fig. 5 plot but for negative strength of impurity $V_{0}=-0.1$.

subbands are partially occupied) does not change its sign as we change the sign of disorder. ${ }^{24}$ Comparing Figs. 7 and 8 , we again see that the hybrid skew scattering is more pronounced for larger impurity strength.

As we increase the disorder by increasing $\gamma_{\text {Born }}$, the skew scattering becomes less important while the other mechanisms, such as intrinsic and side jump, become more important. The intrinsic conductivity only gradually decreases with the disorder because the only effect of disorder on the intrinsic component comes from broadening of Green's functions used in the calculation of the intrinsic component. For repulsive scatterers $\left(V_{0}>0\right)$, the skew scattering has sign opposite to the sign of intrinsic and side-jump contributions in the region $-h<\omega_{F}<h$ (see, e.g., Refs. 22 and 24). This explains the sign change we observed in Figs. 5, 6, and 10 in the region $-h<\omega_{F}<h$ as we increase $\gamma_{\text {Born }}$ (more detailed plots are presented in Appendix G).

The positions of points in which the AHE vanishes can be estimated by comparing the Fermi sea intrinsic term $\sigma_{x y}^{I I}$ with the skew-scattering term in Eq. (46) as those two are the major contributions. Physically, the AHE vanishes because the intrinsic deflection of electrons between the scattering events can be balanced by the skew-scattering events (in the cross-over region between intrinsic and extrinsic mechanisms). As the former does not rely on impurities and the latter does (and changes sign with impurities changing sign),

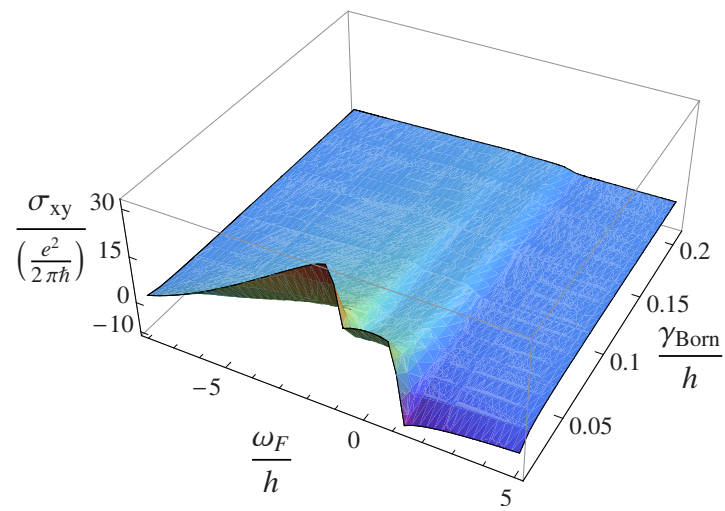

FIG. 8. (Color online) Identical to Fig. 5 plot but for negative strength of impurity $V_{0}=-0.3$. 
we can have full cancellation of the two by choosing the proper sign and strength of impurities.

As it can be seen from Figs. 5-8, the anomalous Hall effect is resonantly increased around the band anticrossing which suggests that for more general band structures, the major contribution to the AHE also comes from the band anticrossings that happened to be in the vicinity of the Fermi level. ${ }^{1}$ This view is well justified for the intrinsic AHE in the metallic regime $\left(\tau \varepsilon_{F} \gg 1\right)$ as it follows from the ThoulessKohmoto-Nightingale-Nijs formula ${ }^{51}$ applied to the AHE. ${ }^{1}$ This leads to the intrinsic AHE conductivity of the order of $e^{2} /(4 \pi \hbar)$ within the region (ii) in Fig. 2. The full conductivity that includes the intrinsic, side-jump, and skew-scattering contributions seems to also have the resonant behavior around the anticrossing for the Rashba model as it follows from our analysis. Whereas our analysis justifies focusing the calculations on simplified phenomenological models near the anticrossing locations, we emphasize that it is unlikely that these would be characterized universally by the Rashba geometry rather than by a combination of Rashba and Dresselhauss symmetry.

In the regime of strong disorder, where this resonant behavior is not pronounced, the disorder broadening of the Green's functions becomes more dominant and the $\sigma_{x y}$ has dependence that can no longer be expanded correctly in powers of $\tau$. This expectation can be easily seen from the expressions for $\sigma_{x y}$ in our formulation or the Kubo formulation, in which

$$
\sigma_{x y} \propto \sum_{\alpha, \beta} \frac{\left\langle\alpha\left|\hat{v}_{x}\right| \beta\right\rangle\left\langle\beta\left|\hat{v}_{y}\right| \alpha\right\rangle}{\left(E_{\alpha}-E_{\beta}\right)^{2}},
$$

where $|\alpha\rangle$ are the exact eigenstates in the presence of disorder and the major contribution for $\sigma_{x y}$ in the dirty limit comes from interband matrix elements. When expanding things in the momentum basis, the denominator is often approximated as $\left[E_{n}(\vec{k})-E_{n^{\prime}}(\vec{k})\right]^{2}+(\hbar / \tau)^{2}$ while the matrix elements are evaluated within the disorder-free eigenstates. Hence, in the limit of large disorder broadening, the denominator is simply replaced by $(\hbar / \tau)^{2}$ and $\sigma_{x y} \sim \tau^{2}$ (this is different for $\sigma_{x x}$ as the contribution from interband matrix elements vanishes and $\left.\sigma_{x x} \sim \tau\right)$. This of course gives an upper bound for the $\sigma_{x y} \sim \tau^{\eta}$ scaling and in intermediate regimes one would expect $\eta$ to be lower than 2 .

In Figs. 9 and 10, we study the AHE calculated in the anticrossing region in order to examine in detail the universal anomalous Hall-effect regimes that could be valid for more general band structures. We now plot in the logarithmic scale $\sigma_{x y}$ as a function of $\sigma_{x x}$ tuned via $n_{i}$ while all other parameters are kept constant. In the clean limit, we recover the skew-scattering behavior $\left[\sigma_{x y} \sim 1 /\left(n_{i} V_{0}\right) \sim \sigma_{x x} / V_{0}\right]$ and our numerical results (bold line) agree well with the analytical results (dashed line) obtained in Sec. III B. In the moderately dirty limit, we observe the intrinsic-side-jump regime $\left(\sigma_{x y}\right.$ $=$ const., this regime is more pronounced for smaller $V_{0}$ ) in which the side-jump and intrinsic mechanisms are dominant. All analytical curves (dashed lines) asymptotically reach this regime when $\sigma_{x x}$ is very small. In the stronger disorder regime, as reported in Ref. 1, the numerical curves have down-

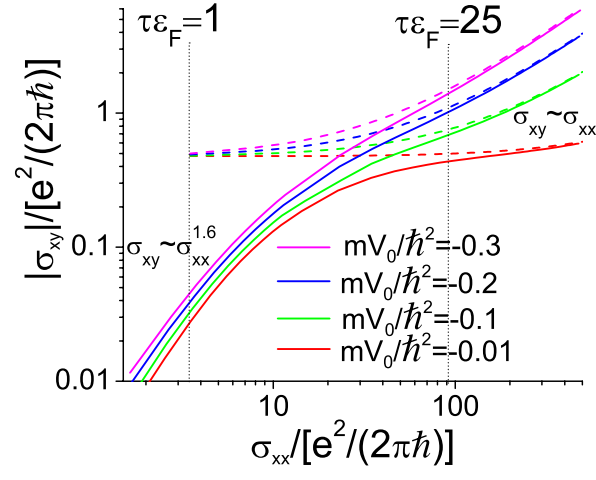

FIG. 9. (Color online) The absolute value of the anomalous Hall conductivity $\left|\sigma_{x y}\right|$ versus the conductivity $\sigma_{x x}$ for the spin-orbit interaction strength $2 \alpha^{2} / h=35.9$. Dimensionality of quantities displayed in this plot is restored.

turn for smaller $\sigma_{x x}$ approaching the third regime in which $\sigma_{x y} \sim \sigma_{x x}^{\varphi}$, with $\varphi \approx 1.6$ in Fig. 9. However, a universal scaling cannot be claimed since for large and positive strength of impurities in Fig. 10 we only observe the reduction of the AHE.

One should keep in mind that the TMA is not fully justified close to the line $\tau \varepsilon_{F}=1$ and our results are meaningful only for $\tau \varepsilon_{F}>1$. Furthermore, since in this regime the resonant behavior is strongly diminished, in realistic threedimensional systems, the result could be more accurately expressed via the averaged matrix elements with some appropriate treatment of the disorder broadening.

Although some experimental works claim to confirm the scaling $\sigma_{x y} \sim \sigma_{x x}^{\varphi}$, with $\varphi$ around $1.6,{ }^{37-41}$ comparison of theory and experiments has to be done with care since determining a scaling exponent over a single decade is often difficult and has led to many errors in the past. For example, in DMS ferromagnets (mentioned in Ref. 1 to support the scaling hypothesis) the change of doping will cause change in the impurity concentration, in the magnetization, and even in the band structure. The theoretical calculations only take into account the change in the impurity concentration and further assume a Rashba symmetry at the crossing points..$^{20,1}$ Note also that within the theoretical treatment, the Hall conductivity changes its sign for repulsive impurities $\left(V_{0}>0\right)$ in Fig.

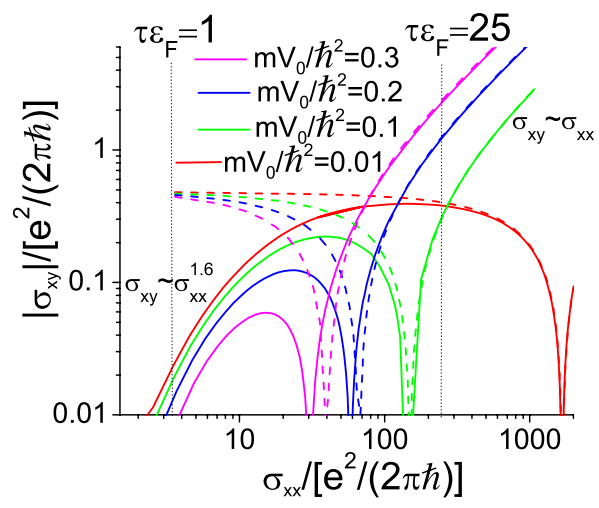

FIG. 10. (Color online) Identical to Fig. 9 plot except for the disorder which is repulsive here $\left(V_{0}>0\right)$. Note that the conductivity $\sigma_{x y}$ changes sign around the cusps. 
10 which is expected as the skew scattering dominating in the clean limit has the sign opposite to the sign of the intrinsic contribution dominating in the dirty limit [see Eq. (46)]. These types of changes of signs have also been observed in experimental systems, e.g., DMS ${ }^{52}$ and of course at that stage scaling is not justified.

\section{AMR IN RASHBA SYSTEMS}

In Sec. III, we showed how the formalism developed in Sec. II can be applied to calculations of the anomalous Hall effect in multiple-band systems on the example of a Rashba system. In this section, we perform calculations of the AMR in 2DEG with the in-plane exchange field, spin-orbit interaction, and magnetic impurities following the same formalism. A general numerical procedure allows us to rigorously perform AMR calculations in multiple-band anisotropic systems. Within the Boltzmann equation approach, such calculations are usually performed by using the relaxation-time approximation in which the transport relaxation time $\tau$ is calculated from the scattering amplitudes without fully taking into account the asymmetries. ${ }^{30,34}$ This approach was improved in Ref. 53 by introducing the perpendicular relaxation time $\tau_{\perp}$. However, in some cases this improvement is yet not sufficient and some of the present authors formulated a procedure for finding an exact solution to the Boltzmann equation in Ref. 35. Here we propose an alternative approach for AMR calculations in multiple-band anisotropic systems to the one proposed in Ref. 35.

We consider here a 2DEG Rashba Hamiltonian with additional in-plane exchange field $h_{x}$ directed along the $x$ axis without any loss of generality

$$
\hat{H}_{R}=\mathbf{k}^{2} / 2+\alpha \mathbf{k} \cdot \hat{\boldsymbol{\sigma}} \times \mathbf{z}-h_{x} \hat{\sigma}_{x}-h \hat{\sigma}_{z}+\hat{V}(\mathbf{r}),
$$

where now $\hat{V}(\mathbf{r})$ describes the disorder corresponding to dilute charged magnetic impurities ${ }^{34,54,55}$

$$
\hat{V}(\mathbf{r})=V_{0}\left(a \hat{\sigma}_{0}+\hat{\sigma}_{x}\right) \sum_{i} \delta\left(\mathbf{r}-\mathbf{r}_{i}\right),
$$

where $\mathbf{r}_{i}$ describes the positions of random impurities and we assume that the magnetic impurities are magnetized along the exchange field. The quantity $a$ describes the relative strength of the electric part of impurity with respect to the magnetic part. Note that the AMR is measured by changing the direction of electric field $\mathbf{E}$ which is equivalent to changing the direction of the exchange field.

For the AMR, we only need the diagonal conductivities, thus the Fermi sea contribution given by Eq. (43) vanishes. The AMR can be calculated from Eq. (42) and we only need to calculate Green's functions at the Fermi level. We calculate $\hat{\Sigma}_{e q}^{R, A}$ and Green's functions $\hat{G}_{e q}^{R, A}$ using the self-consistent TMA

$$
\begin{gathered}
\hat{T}_{e q}^{R, A}=V_{0}\left(\hat{1}-V_{0} \hat{\gamma}^{R, A}\right)^{-1}, \\
\hat{\Sigma}_{e q}^{R, A}=n_{i} \hat{T}_{e q}^{R, A}\left(\omega_{F}\right)=\Sigma_{e q 0}^{R, A} \hat{\sigma}_{0}+\Sigma_{e q \mathrm{x}}^{R, A} \hat{\sigma}_{x}+\Sigma_{e q \mathrm{z}}^{R, A} \hat{\sigma}_{z},
\end{gathered}
$$

$$
\begin{aligned}
& \hat{G}_{e q}^{R}=\left(\omega \hat{1}-\hat{H}_{0}-\hat{\Sigma}_{e q}^{R}\right)^{-1} \\
&= \frac{\left(W-\frac{k^{2}}{2}\right) \hat{\sigma}_{0}+\alpha k_{y} \hat{\sigma}_{x}-\alpha k_{x} \hat{\sigma}_{y}-H \hat{\sigma}_{z}}{\left(W-\frac{k^{2}}{2}\right)^{2}-H^{2}-\alpha^{2} k^{2}+2 H_{x} \alpha k_{y}} \\
&-\frac{H_{x} \hat{\sigma}_{x}}{\left(W-\frac{k^{2}}{2}\right)^{2}-H^{2}-\alpha^{2} k^{2}+2 H_{x} \alpha k_{y}}, \\
& \hat{G}_{e q}^{A}=\left(\hat{G}_{e q}^{R}\right)^{\dagger},
\end{aligned}
$$

where $W=\omega-\Sigma_{e q 0}^{R}, \quad H=h-\Sigma_{e q \mathrm{Z}}^{R}, \quad H_{x}=h_{x}-\Sigma_{e q \mathrm{X}}^{R}, \quad$ and $\hat{\gamma}^{R, A}$ $=\int d^{2} k /(2 \pi)^{2} \hat{G}_{e q}^{R, A}(\mathbf{k}, \omega) \equiv \gamma^{R, A} \hat{\sigma}_{0}+\gamma_{x}^{R, A} \hat{\sigma}_{x}+\gamma_{z}^{R, A} \hat{\sigma}_{z}$. We calculate the self-consistent value of the self-energy $\hat{\Sigma}_{e q}^{R, A}\left(\omega_{F}\right)$ by iterating Eq. (52) until the prescribed accuracy is reached.

As soon as we know the $T$ matrix, we can substitute it into Eq. (40) and find the matrix $\hat{\rho}$ by performing the momentum integrations in the right-hand side. Finally, by substituting $\hat{\rho}$ into Eq. (42) we can calculate the conductivity. Note that throughout this section, the angular part of the momentum integrations is calculated analytically while the radial part is calculated numerically. The anisotropic resistance in our system is defined as follows:

$$
\mathrm{AMR}=-\frac{\sigma_{x x}-\sigma_{y y}}{\sigma_{x x}+\sigma_{y y}},
$$

and it describes the relative difference in conductivity for current flowing parallel or perpendicular to the magnetization (represented by the exchange field and/or impurity magnetization).

First, we calculate the anisotropic magnetoresistance in Rashba system with in-plane exchange field and nonmagnetic delta scatterers [see Eq. (30), the magnetic scatterers are absent in this model]. Kato et al. ${ }^{36}$ found vanishing AMR in the regime (i) (see Fig. 2) when both subbands are partially occupied due to the cancellation of the nonvertex and vertex parts in the Kubo formulation. In Fig. 11, we observe the nonvanishing AMR in the regime (i) and this suggests the importance of the higher order diagrams [such as plotted in Fig. 4(e)] not only for the AHE but also for the AMR. The AMR effect resulting from the higher order diagrams is more pronounced for the larger strength of impurities, similar to the AHE. The AMR approaches its maximum around the point at which the exchange energy is comparable to the spin-orbit energy, $2 h_{x} \sim \alpha$. We note that the nonzero but comparatively weak magnitude of the AMR here in the Rashba system is reminiscent of the results in three-dimensional DMS ferromagnets. ${ }^{34}$ This agrees with physical intuition. Under comparison of two mechanisms by which AMR can arise-carrier polarization/anisotropy in wave functions and impurity polarization/anisotropy in scattering operator (see Fig. 1 of Ref. 34) - the former implies a competition between the exchange and spin-orbit terms (in the Hamiltonian) resulting in reduced anisotropy strength. 


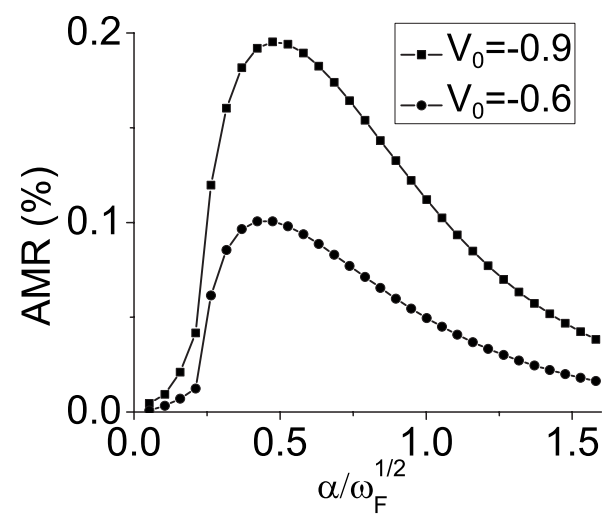

FIG. 11. The AMR $(100 \%$ corresponds to $\mathrm{AMR}=1)$ as a function of the dimensionless spin-orbit interaction strength $\alpha / \sqrt{\omega_{F}}\left(\omega_{F}\right.$ is counted from the middle of the gap in Fig. 2). The parameters of the model are $V_{0}=-0.6$ and $-0.9, n_{i} / \omega_{F}=0.01$, and $h_{x} / \omega_{F}=0.3$.

Consequently, even though observation of the AMR effect is deemable in the absence of magnetic scatterers, we expect much more pronounced effects when the magnetic scatterers are present. Our numerical results in Fig. 12 (plotted together with the analytical results from Ref. 35) confirm this. For the case when the Fermi level crosses only one band [region (ii) in Fig. 2], it was found in Ref. 35 that $\mathrm{AMR}=1 /\left(2-a^{2}\right)$ when $|a|<1$ and $\mathrm{AMR}=1 / a^{2}$ when $|a|>1$, provided the exchange fields are small. For the case when the Fermi level crosses two bands [region (i) in Fig. 2] it was found in Ref. 35 that $\mathrm{AMR}=a^{2}$ when $|a|<1$ and $\mathrm{AMR}=1 / a^{2}$ when $|a|$ $>1$, in the limit of large Fermi energy (compared to the spin-orbit and exchange splittings). We observe a perfect agreement between our numerical results and the analytical results from Ref. 35. The result in Fig. 12(a) cannot be reproduced within the common approximate approaches ${ }^{30,34,53}$ based on the relaxation-time approximation as it was pointed out in Ref. 35. The nonphysical divergence in $\sigma_{y y}$ at the point $a=1$ in Fig. 12 is caused by the special choice of the scat-

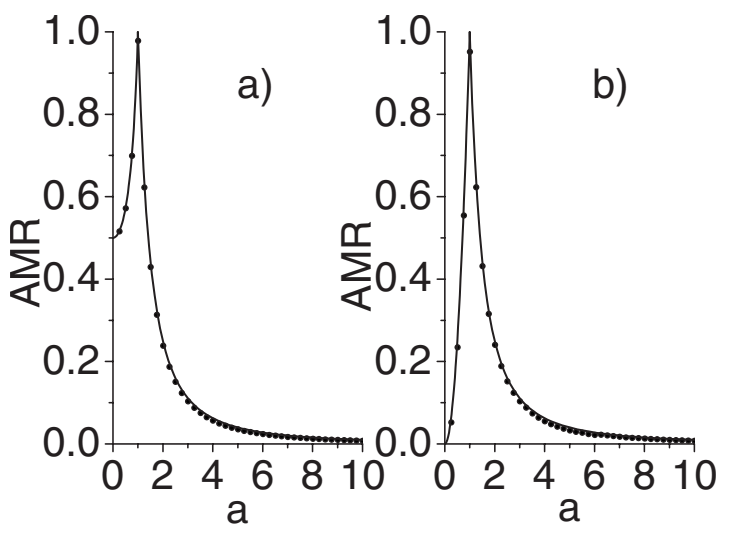

FIG. 12. The AMR as a function of the relative strength $a$ of the electric and magnetic parts of impurity potential. By solid line we plot analytical results and dots represent numerical results: (a) Fermi level crosses only one band $\left(\omega_{F}=0\right)$ with the following dimensionless parameters: $V_{0}=0.05, \alpha=1.4, n_{i}=0.0015, h_{x}=0.0015$, and $h=0.015$; (b) Fermi level crosses both bands $V_{0}=0.05, \alpha$ $=0.03, n_{i}=0.002, h_{x}=0.002$, and $h=0.001$. tering potential. ${ }^{35}$ As soon as the spatial dependences of the electric and magnetic parts cease to be identical [in Eq. (50), they correspond both to delta scatterers] the divergence of the $\sigma_{y y}$ is removed (causing $\mathrm{AMR}<1$ ).

\section{CONCLUSIONS}

We have developed a framework for transport calculations in multiple-band noninteracting Fermi systems. By applying this framework to Rashba 2DEG, we have resolved some recent discrepancies related to the AHE in such systems. The findings of this simple 2D model have been linked to higher dimensional systems arguing that most likely the major contributions to the AHE come from the band anticrossing regions similar to one observed in the Rashba model. Our analytical and numerical results reveal the crossover between the skew-scattering-dominated regime in clean systems $\left(\sigma_{x y}\right.$ $\left.\sim V_{0} / \gamma_{\text {Born }} \sim \sigma_{x x}\right)$ and the intrinsic deflection dominated regime in moderately dirty systems $\left(\sigma_{x y} \sim\right.$ const. $)$. In dirty systems, we observe the third distinct regime also dominated by the intrinsic contribution. In this regime, the AHE diminishes in a manner similar to $\sigma_{x y} \sim \sigma_{x x}^{\varphi}$, with $\varphi$ being close to 1.6. This, however, cannot be called by scaling as the theory is not meaningful in a sufficiently wide range of $\sigma_{x y}$ and $\sigma_{x x}$ due to breakdown of the TMA when $\tau \varepsilon_{F} \sim 1$. For the repulsive impurities, we observe that the intrinsic and skew anomalous Hall effects have opposite signs. As a result, the crossover between those two is also accompanied by the change of sign of the AHE. We suggest to engineer samples with repulsive impurities in order to see this change of sign in the AHE and a possible effect on the scaling.

We have resolved some discrepancies between the AHE results obtained by using the Keldysh, Kubo, and Boltzmann approaches by considering the higher order skew-scattering processes. We have included similar higher order processes in our AMR calculations and shown their importance for the Rashba model in which nonvertex and vertex diagrammatic parts cancel each other. We have calculated the AMR in anisotropic systems properly taking into account the anisotropy of the nonequilibrium distribution function. These calculations confirm recent findings on the unreliability of common approximate approaches to the Boltzmann equation.

\section{ACKNOWLEDGMENTS}

We gratefully acknowledge fruitful discussions with E. I. Rashba, V. Dugaev, J. Inoue, T. Jungwirth, A. H. MacDonald, G. E. W. Bauer, N. Nagaosa, and S. Onoda. This work was supported by the Alfred P. Sloan Foundation (Y.T.), by ONR under Grant No. ONR-N000140610122, by NSF under Grant No. DMR-0547875, by SWAN-NRI and Czech Grants No. KJB100100802, No. LC510, and No. AV0Z10100521. J.S. is a Cottrell Scholar of the Research Corporation.

\section{APPENDIX A: GENERALIZATIONS FOR SHORT-RANGE DISORDER}

In Sec. II A, we derive the kinetic equation with the selfenergy expression that is valid for uniform systems. Here, we 
generalize this self-energy to nonuniform systems in the presence of a short-range disorder postulated by the following infinite set of correlators:

$$
\begin{gathered}
\langle V V\rangle=\langle V\rangle\langle V\rangle+V_{2} \delta_{\mathbf{r}_{1}-\mathbf{r}_{2}}, \\
\langle V V V\rangle=\sum\langle V V\rangle\langle V\rangle+V_{3} \delta_{\mathbf{r}_{1}-\mathbf{r}_{3}}, \\
\langle V V V V\rangle=\sum(\langle V V V\rangle\langle V\rangle+\langle V V\rangle\langle V V\rangle)+V_{4} \delta_{\mathbf{r}_{1}-\mathbf{r}_{4}}, \text { etc. }
\end{gathered}
$$

where we decouple the correlators into a product of two lower order correlators and sum all such products, $\delta_{\mathbf{r}_{1}-r_{N}}$ $=\prod_{i=1 . . N-1} \delta\left(\mathbf{r}_{i}-\mathbf{r}_{i+1}\right)$. Note that usually the averaged impurity potential is zero, $\langle V\rangle=V_{1}=0$.

After performing the averaging procedure for the Green's function, we again arrive at the kinetic Eq. (6) with the selfenergy given by the following formal expression:

$$
\check{\Sigma}=\left.\left(\check{V}_{0}+\check{V}_{0} \otimes \check{G} \otimes \check{V}_{0}+\ldots\right)\right|_{V_{0}^{n} \rightarrow V_{n}},
$$

where $\check{V}_{0}=V_{0}\left(\begin{array}{cc}\hat{\eta} & 0 \\ 0 & \hat{\eta}\end{array}\right) \delta\left(1-1^{\prime}\right)$ and in the term of $n$th order proportional to $V_{0}^{n}$ we replace $V_{0}^{n}$ by $V_{n}$ which ensures that the correlators in Eq. (A1) are properly considered. It is convenient to introduce the notation

$$
\check{E}=\left(\check{V}_{0}+\check{V}_{0} \otimes \check{G} \otimes \check{V}_{0}+\cdots\right),
$$

which, in analogy with the self-energy, also has retarded (advanced) $E^{R, A}$ and lesser $E^{<}$components. Equation (A3) can be rewritten in the form of $T$-matrix equation, $\check{E}=\check{V} \otimes[\check{1}$ $+\check{G} \otimes \check{E}]$, which leads to the expressions for the self-energies

$$
\begin{gathered}
\hat{\Sigma}^{R, A}=\left.\hat{E}^{R, A}\right|_{V_{0}^{n} \rightarrow V_{n},}, \\
\hat{\Sigma}^{<}=\left.\left(\hat{E}^{R} \otimes \hat{G}^{<} \otimes \hat{E}^{A}\right)\right|_{V_{0}^{n} \rightarrow V_{n}},
\end{gathered}
$$

where the notation $\left.\right|_{V_{0}^{n} \rightarrow V_{n}}$ is formal and it means that $\hat{E}^{R, A}$ has to be fist expanded with respect to $V_{0}$ and then after grouping $V_{0}$ 's together the substitution has to be applied. Equations (14) and (15) can now be rederived for nonuniform systems with the disorder given by Eq. (A1) and $\hat{\Sigma}_{1}^{<}$ $=\left.\left(\hat{E}^{R} \otimes \hat{G}_{1}^{<} \otimes \hat{E}^{A}\right)\right|_{V_{0}^{n} \rightarrow V_{n}}$.

Nevertheless, for the purposes of this paper, it is sufficient to consider the uniform and stationary case. This leads to substantial simplifications outlined in Secs. II B and II C. Results of Secs. II B and II C also hold for the disorder given by Eq. (A1) with the exception of Eqs. (20) and (28) that should be replaced by the following equations:

$$
\begin{aligned}
\hat{\Sigma}_{1}^{<} & =\left[\hat{E}_{e q}^{R}\left(\int \frac{d^{2} k^{\prime}}{(2 \pi)^{2}} \hat{G}_{1}^{<}\left(\mathbf{k}^{\prime}\right)\right) \hat{E}_{e q}^{A}\right]_{V_{0}^{n} \rightarrow V_{n}}, \\
\hat{\Sigma}_{\mathbf{E}}^{R, A} & =\left[\hat{E}_{e q}^{R, A}\left(\int \frac{d^{2} k^{\prime}}{(2 \pi)^{2}} \hat{G}_{\mathbf{E}}^{R, A}\left(\mathbf{k}^{\prime}\right)\right) \hat{E}_{e q}^{R, A}\right]_{V_{0}^{n} \rightarrow V_{n}} .
\end{aligned}
$$

Finally, we would like to present several examples in which the disorder given by Eq. (A1) is realized. The simplest example is given by Eq. (30) and in this case $V_{n}$ $=n_{i} V_{0}^{n}$. For the disorder given by Eq. (44), we have $V_{n}$ $=n_{i}\left\langle\left(V_{0}^{i}\right)^{n}\right\rangle_{d i s}$. For the Gaussian white-noise disorder, only $V_{2}$ is nonzero and $\left.V_{n}\right|_{n \neq 2}=0$. For the telegraph white-noise disorder all odd correlators vanish, $V_{2 n+1}=0$.

\section{APPENDIX B: CALCULATION OF SELF-CONSISTENT SELF-ENERGY $\hat{\mathbf{\Sigma}}_{e q}^{R, A}$}

The following relations can be calculated by a direct analytical integration of Eq. (34):

$$
\begin{gathered}
\hat{\gamma}^{R, A}=\int d^{2} k /(2 \pi)^{2} \hat{G}_{e q}^{R, A}(\mathbf{k}, \omega) \equiv \gamma^{R, A} \hat{\sigma}_{0}+\gamma_{z}^{R, A} \hat{\sigma}_{z}, \\
\gamma^{R}=\frac{\left(K_{+}-2 W\right)\left[\ln \left(K_{0}-K_{+}\right)-\ln \left(-K_{+}\right)\right]}{2 \pi\left(K_{-}-K_{+}\right)} \\
-\frac{\left(K_{-}-2 W\right)\left[\ln \left(K_{0}-K_{-}\right)-\ln \left(-K_{-}\right)\right]}{2 \pi\left(K_{-}-K_{+}\right)}, \\
\gamma_{z}^{R}=\frac{\ln \left(K_{0}-K_{+}\right)-\ln \left(-K_{+}\right)}{\pi\left(K_{-}-K_{+}\right) / H}-\frac{\ln \left(K_{0}-K_{-}\right)-\ln \left(-K_{-}\right)}{\pi\left(K_{-}-K_{+}\right) / H}, \\
\gamma^{A}=\left(\gamma^{R}\right)^{*} ; \gamma_{x(y, z)}^{A}=\left(\gamma_{x(y, z)}^{R}\right) ; \gamma_{y(z)}^{R}=0,
\end{gathered}
$$

where $\quad W=\omega-\Sigma_{\text {eq } 0}^{R}, \quad H=h-\Sigma_{\text {eqz }}^{R}, \quad K_{ \pm}=2(W$ $+\alpha^{2} \mp \sqrt{\left.H^{2}+2 W \alpha^{2}+\alpha^{4}\right)}$, and $K_{0}=k_{0}^{2}$ describes the cutoff $k_{0}$ in momentum integration.

For each energy, $\Sigma_{e q 0}^{R}(\omega)$ and $\Sigma_{e q z}^{R}(\omega)$ are calculated by performing a number of iterations with the consequent iteration according to

$$
\begin{aligned}
& \Sigma_{e q 0}^{R, A}=\frac{1}{2} \operatorname{Tr}\left[n_{i} V_{0}\left(\hat{1}-V_{0} \hat{\gamma}^{R, A}\right)^{-1} \hat{\sigma}_{0}\right], \\
& \sum_{e q z}^{R, A}=\frac{1}{2} \operatorname{Tr}\left[n_{i} V_{0}\left(\hat{1}-V_{0} \hat{\gamma}^{R, A}\right)^{-1} \hat{\sigma}_{z}\right] .
\end{aligned}
$$

The iterations are performed until the prescribed accuracy is reached.

\section{APPENDIX C: CALCULATION OF THE MATRIX $\hat{\rho}(\omega)$}

For the electric field $\mathbf{E}$ along the $y$ axis $\mathbf{E}=\left(0, E_{y}\right)$, we solve here the linear Eq. (40) for the elements of the matrix $\hat{\rho}(\omega)$ by performing analytically the momentum integrations of the Green's functions $\hat{G}_{e q}^{R, A}(\mathbf{k}, \omega)$ [given by Eq. (34)] in the right-hand side. For each energy $\omega$, we obtain the following expressions that also depend on the self-consistent values of $\sum_{00}^{R}(\omega)$ and $\Sigma_{0 z}^{R}(\omega)$ :

$$
\rho_{--}(\omega)=\rho_{++}(\omega)=0,
$$




$$
\begin{aligned}
& \rho_{+-}(\omega)=i \partial_{\omega} n_{F} e E_{y} \alpha\left\{\frac{\left[K_{-}^{2}+4(H+W)\left(H^{*}-W^{*}\right)\right] \ln \left(-K_{-}\right)}{\left(K_{-}-K_{-}^{*}\right)\left(K_{-}-K_{+}\right)\left(K_{-}-K_{+}^{*}\right) \pi}-\frac{\left[K_{-}^{* 2}+4(H+W)\left(H^{*}-W^{*}\right)\right] \ln \left(-K_{-}^{*}\right)}{\left(K_{-}-K_{-}^{*}\right)\left(K_{-}^{*}-K_{+}\right)\left(K_{-}^{*}-K_{+}^{*}\right) \pi}\right. \\
& \left.+\frac{\left[K_{+}^{2}+4(H+W)\left(H^{*}-W^{*}\right)\right] \ln \left(-K_{+}\right)}{\left(K_{-}-K_{+}\right)\left(K_{-}^{*}-K_{+}\right)\left(K_{+}-K_{+}^{*}\right) \pi}-\frac{\left[K_{+}^{* 2}+4(H+W)\left(H^{*}-W^{*}\right)\right] \ln \left(-K_{+}^{*}\right)}{\left(K_{-}-K_{+}^{*}\right)\left(K_{-}^{*}-K_{+}^{*}\right)\left(K_{+}-K_{+}^{*}\right) \pi}\right\} \\
& \times\left\{-1+\left[\frac{\left(2 H-K_{-}+2 W\right)\left(2 H^{*}+K_{-}-2 W^{*}\right) \ln \left(-K_{-}\right)}{\left(K_{-}-K_{-}^{*}\right)\left(K_{-}-K_{+}\right)\left(K_{-}-K_{+}^{*}\right) \pi}-\frac{\left(2 H-K_{-}^{*}+2 W\right)\left(2 H^{*}+K_{-}^{*}-2 W^{*}\right) \ln \left(-K_{-}^{*}\right)}{\left(K_{-}-K_{-}^{*}\right)\left(K_{-}^{*}-K_{+}\right)\left(K_{-}^{*}-K_{+}^{*}\right) \pi}\right.\right. \\
& \left.\left.+\frac{\left(2 H-K_{+}+2 W\right)\left(2 H^{*}+K_{+}-2 W^{*}\right) \ln \left(-K_{+}\right)}{\left(K_{-}-K_{+}\right)\left(K_{-}^{*}-K_{+}\right)\left(K_{+}-K_{+}^{*}\right) \pi}-\frac{\left(2 H-K_{+}^{*}+2 W\right)\left(2 H^{*}+K_{+}^{*}-2 W^{*}\right) \ln \left(-K_{+}^{*}\right)}{\left(K_{-}-K_{+}^{*}\right)\left(K_{-}^{*}-K_{+}^{*}\right)\left(K_{+}-K_{+}^{*}\right) \pi}\right] n_{i} T_{++} T_{--}^{*}\right\}^{-1} \text {, } \\
& \rho_{-+}(\omega)=i \partial_{\omega} n_{F} e E_{y} \alpha\left\{\frac{\left[K_{-}^{2}+4(H-W)\left(H^{*}+W^{*}\right)\right] \ln \left(-K_{-}\right)}{\left(K_{-}-K_{-}^{*}\right)\left(K_{-}-K_{+}\right)\left(K_{-}-K_{+}^{*}\right) \pi}-\frac{\left[K_{-}^{* 2}+4(H-W)\left(H^{*}+W^{*}\right)\right] \ln \left(-K_{-}^{*}\right)}{\left(K_{-}-K_{-}^{*}\right)\left(K_{-}^{*}-K_{+}\right)\left(K_{-}^{*}-K_{+}^{*}\right) \pi}\right. \\
& \left.+\frac{\left[K_{+}^{2}+4(H-W)\left(H^{*}+W^{*}\right)\right] \ln \left(-K_{+}\right)}{\left(K_{-}-K_{+}\right)\left(K_{-}^{*}-K_{+}\right)\left(K_{+}-K_{+}^{*}\right) \pi}-\frac{\left[K_{+}^{* 2}+4(H-W)\left(H^{*}+W^{*}\right)\right] \ln \left(-K_{+}^{*}\right)}{\left(K_{-}-K_{+}^{*}\right)\left(K_{-}^{*}-K_{+}^{*}\right)\left(K_{+}-K_{+}^{*}\right) \pi}\right\} \\
& \times\left\{-1+\left[\frac{\left(2 H+K_{-}-2 W\right)\left(2 H^{*}-K_{-}+2 W^{*}\right) \ln \left(-K_{-}\right)}{\left(K_{-}-K_{-}^{*}\right)\left(K_{-}-K_{+}\right)\left(K_{-}-K_{+}^{*}\right) \pi}-\frac{\left(2 H+K_{-}^{*}-2 W\right)\left(2 H^{*}-K_{-}^{*}+2 W^{*}\right) \ln \left(-K_{-}^{*}\right)}{\left(K_{-}-K_{-}^{*}\right)\left(K_{-}^{*}-K_{+}\right)\left(K_{-}^{*}-K_{+}^{*}\right) \pi}\right.\right. \\
& \left.\left.+\frac{\left(2 H+K_{+}-2 W\right)\left(2 H^{*}-K_{+}+2 W^{*}\right) \ln \left(-K_{+}\right)}{\left(K_{-}-K_{+}\right)\left(K_{-}^{*}-K_{+}\right)\left(K_{+}-K_{+}^{*}\right) \pi}-\frac{\left(2 H+K_{+}^{*}-2 W\right)\left(2 H^{*}-K_{+}^{*}+2 W^{*}\right) \ln \left(-K_{+}^{*}\right)}{\left(K_{-}-K_{+}^{*}\right)\left(K_{-}^{*}-K_{+}^{*}\right)\left(K_{+}-K_{+}^{*}\right) \pi}\right] n_{i} T_{--} T_{++}^{*}\right\}^{-1} \text {, }
\end{aligned}
$$

where $T_{ \pm \pm}$corresponds to the elements of the matrix $\hat{T}_{e q}^{R}(\omega)$.

\section{APPENDIX D: CALCULATION OF THE MATRIX $\hat{\rho}_{\mathrm{E}}^{R}(\omega)$}

For the electric field $\mathbf{E}$ along the $y$ axis $\mathbf{E}=\left(0, E_{y}\right)$, we solve here the linear Eq. (41) for the elements of the matrix $\hat{\rho}_{\mathbf{E}}^{R, A}(\omega)$ by performing analytically the momentum integrations of the Green's functions $\hat{G}_{e q}^{R, A}(\mathbf{k}, \omega)$ [given by Eq. (34)] in the righthand side. For each energy $\omega$, we obtain the following expressions that also depend on the self-consistent values of $\Sigma_{00}^{R}(\omega)$ and $\Sigma_{0 z}^{R}(\omega)$ :

$$
\begin{gathered}
\rho_{E_{y}--}^{R}(\omega)=\rho_{E_{y^{++}}}^{R}(\omega)=0, \\
\rho_{E_{y}^{+-}}^{R}(\omega)=4 i \alpha\left[H\left(-1+\partial_{\omega} \Sigma_{00}^{R}\right)-W \partial_{\omega} \Sigma_{0 z}^{R}\right]\left\{K_{-}^{2}-K_{+}^{2}+2 K_{-} K_{+}\left[-\ln \left(-K_{-}\right)+\ln \left(-K_{+}\right)\right]\right\} \\
\times\left\{( K _ { - } - K _ { + } ) \left[K_{-}^{3} K_{+} \pi+K_{+}^{3} K_{-} \pi+K_{-}^{2} K_{+} n_{i} T_{--} T_{++}-4 K_{+} n_{i} T_{--} T_{++}\left(H^{2}-W^{2}\right)+K_{+}^{2} K_{-} n_{i} T_{--} T_{++}-4 K_{-} n_{i} T_{--} T_{++}\left(H^{2}-W^{2}\right)\right.\right. \\
\left.\left.-2 \pi K_{+}^{2} K_{-}^{2}-8 K_{+} K_{-} n_{i} T_{--} T_{++} W\right]+2 K_{-} K_{+} n_{i} T_{--} T_{++}\left[4 H^{2}+\left(K_{-}-2 W\right)\left(-K_{+}+2 W\right)\right]\left[\ln \left(-K_{-}\right)-\ln \left(-K_{+}\right)\right]\right\}^{-1}, \\
\rho_{E_{y}{ }^{-+}}^{R}(\omega)=-\rho_{E_{y^{+}}}^{R}(\omega),
\end{gathered}
$$

where $T_{ \pm \pm}$corresponds to the elements of the matrix $\hat{T}_{e q}^{R}(\omega)$.

\section{APPENDIX E: CALCULATION OF THE FERMI-SURFACE CONDUCTIVITY}

For the electric field $\mathbf{E}$ along the $y$ axis $\mathbf{E}=\left(0, E_{y}\right)$, we perform momentum $k$ and frequency $\omega$ integrations in Eq. (42). It is convenient to divide the resultant conductivity into two parts: the bare bubble part $\sigma_{x y(y))}^{J b}$ that corresponds to calculating only 
the second line in Eq. (42) effectively assuming that $\hat{\rho}\left(\omega_{F}\right)=0$ and self-consistent part $\sigma_{x y(y y)}^{I s c}$ that corresponds to calculating the first line in Eq. (42) that takes into account correction due to self-consistent calculation of $\hat{\rho}\left(\omega_{F}\right)$,

$$
\begin{aligned}
& \sigma_{x y}^{I}=\sigma_{x y}^{I b}+\sigma_{x y}^{I s c}, \\
& \sigma_{y y}^{I}=\sigma_{y y}^{I b}+\sigma_{y y}^{I s c} .
\end{aligned}
$$

We arrive at analytical expressions for the bare bubble contributions to the conductivities $\sigma_{x y}^{I b}$ and $\sigma_{y y}^{I b}$ that depend on the self-consistent values of $\Sigma_{00}^{R}\left(\omega_{F}\right)$ and $\Sigma_{0 z}^{R}\left(\omega_{F}\right)$ at the Fermi surface

$$
\begin{aligned}
& \frac{\sigma_{x y}^{I b}}{e^{2} / \hbar}=-\frac{2 i \alpha^{2}\left[-H^{*}\left(K_{-}+2 W\right)+H\left(K_{-}+2 W^{*}\right)\right] \ln \left(-K_{-}\right)}{\left(K_{-}-K_{-}^{*}\right)\left(K_{-}-K_{+}\right)\left(K_{-}-K_{+}^{*}\right) \pi^{2}}-\frac{2 i \alpha^{2}\left[H^{*}\left(K_{-}^{*}+2 W\right)-H\left(K_{-}^{*}+2 W^{*}\right)\right] \ln \left(-K_{-}^{*}\right)}{\left(K_{-}-K_{-}^{*}\right)\left(K_{-}^{*}-K_{+}\right)\left(K_{-}^{*}-K_{+}^{*}\right) \pi^{2}} \\
& -\frac{2 i \alpha^{2}\left[-H^{*}\left(K_{+}+2 W\right)+H\left(K_{+}+2 W^{*}\right)\right] \ln \left(-K_{+}\right)}{\left(K_{-}-K_{+}\right)\left(K_{-}^{*}-K_{+}\right)\left(K_{+}-K_{+}^{*}\right) \pi^{2}}-\frac{2 i \alpha^{2}\left[H^{*}\left(K_{+}^{*}+2 W\right)-H\left(K_{+}^{*}+2 W^{*}\right)\right] \ln \left(-K_{+}^{*}\right)}{\left(K_{-}-K_{+}^{*}\right)\left(K_{-}^{*}-K_{+}^{*}\right)\left(K_{+}-K_{+}^{*}\right) \pi^{2}}, \\
& \frac{\sigma_{y y}^{l b}}{e^{2} / \hbar}=-\frac{K_{-}^{2}\left(K_{-}-3 K_{+}\right) K_{+}^{*} \ln \left(-K_{-}\right)}{4\left(K_{-}-K_{+}\right)^{3}\left(K_{-}-K_{+}^{*}\right) \pi^{2}}-\frac{K_{-}^{3}\left[K_{-}\left(K_{-}+K_{-}^{*}\right)-\left(K_{-}+3 K_{-}^{*}\right) K_{+}+2 K_{+}^{2}\right] \ln \left(-K_{-}\right)}{4\left(K_{-}-K_{-}^{*}\right)\left(K_{-}-K_{+}\right)^{3}\left(K_{-}-K_{+}^{*}\right) \pi^{2}}+\frac{K_{-} K_{-}^{* 2}\left(K_{-}^{*}-3 K_{+}^{*}\right) \ln \left(-K_{-}^{*}\right)}{4\left(K_{-}-K_{-}^{*}\right)\left(K_{-}^{*}-K_{+}^{*}\right)^{3} \pi^{2}} \\
& +\frac{K_{-}^{* 3}\left[K_{-}^{*}\left(K_{-}^{*}+K_{+}\right)-\left(K_{-}^{*}+3 K_{+}\right) K_{+}^{*}+2 K_{+}^{* 2}\right] \ln \left(-K_{-}^{*}\right)}{4\left(K_{-}-K_{-}^{*}\right)\left(K_{-}^{*}-K_{+}\right)\left(K_{-}^{*}-K_{+}^{*}\right)^{3} \pi^{2}}-\frac{K_{+}^{2}\left(3 K_{-}-K_{+}\right) K_{+}^{*} \ln \left(-K_{+}\right)}{4\left(K_{-}-K_{+}\right)^{3}\left(K_{+}-K_{+}^{*}\right) \pi^{2}} \\
& -\frac{K_{+}^{3}\left[2 K_{-}^{2}+K_{+}\left(K_{-}^{*}+K_{+}\right)-K_{-}\left(3 K_{-}^{*}+K_{+}\right)\right] \ln \left(-K_{+}\right)}{4\left(K_{-}^{*}-K_{+}\right)\left(K_{-}-K_{+}\right)^{3}\left(K_{+}-K_{+}^{*}\right) \pi^{2}}+\frac{K_{-} K_{+}^{* 2}\left(3 K_{-}^{*}-K_{+}^{*}\right) \ln \left(-K_{+}^{*}\right)}{4\left(K_{-}-K_{+}^{*}\right)\left(K_{-}^{*}-K_{+}^{*}\right)^{3} \pi^{2}} \\
& +\frac{K_{+}^{* 3}\left[2 K_{-}^{* 2}+K_{+}^{*}\left(K_{+}+K_{+}^{*}\right)-K_{-}^{*}\left(3 K_{+}+K_{+}^{*}\right)\right] \ln \left(-K_{+}^{*}\right)}{4\left(K_{-}-K_{+}^{*}\right)\left(K_{-}^{*}-K_{+}^{*}\right)^{3}\left(K_{+}-K_{+}^{*}\right) \pi^{2}} \\
& -\frac{K_{-} K_{+}\left(K_{-}^{* 2}+K_{+}^{* 2}\right)-K_{-}^{2}\left(K_{-}^{* 2}-K_{-}^{*} K_{+}^{*}+K_{+}^{* 2}\right)-K_{+}^{2}\left(K_{-}^{* 2}-K_{-}^{*} K_{+}^{*}+K_{+}^{* 2}\right)}{2\left(K_{-}-K_{+}\right)^{2}\left(K_{-}^{*}-K_{+}^{*}\right)^{2} \pi^{2}} \\
& -\frac{\left\{4 W W^{*} \alpha^{2}+2 H H^{*}\left(K_{-}-2 \alpha^{2}\right)-K_{-}^{2}\left(W+W^{*}+\alpha^{2}\right)+2 K_{-}\left[W W^{*}+\left(W+W^{*}\right) \alpha^{2}\right]\right\} \ln \left(-K_{-}\right)}{\left(K_{-}-K_{-}^{*}\right)\left(K_{-}-K_{+}\right)\left(K_{-}-K_{+}^{*}\right) \pi^{2}} \\
& +\frac{\left\{4 W W^{*} \alpha^{2}+2 H H^{*}\left(K_{-}^{*}-2 \alpha^{2}\right)-K_{-}^{* 2}\left(W+W^{*}+\alpha^{2}\right)+2 K_{-}^{*}\left[W W^{*}+\left(W+W^{*}\right) \alpha^{2}\right]\right\} \ln \left(-K_{-}^{*}\right)}{\left(K_{-}-K_{-}^{*}\right)\left(K_{-}^{*}-K_{+}\right)\left(K_{-}^{*}-K_{+}^{*}\right) \pi^{2}} \\
& -\frac{\left\{4 W W^{*} \alpha^{2}+2 H H^{*}\left(K_{+}-2 \alpha^{2}\right)-K_{+}^{2}\left(W+W^{*}+\alpha^{2}\right)+2 K_{+}\left[W W^{*}+\left(W+W^{*}\right) \alpha^{2}\right]\right\} \ln \left(-K_{+}\right)}{\left(K_{-}-K_{+}\right)\left(K_{-}^{*}-K_{+}\right)\left(K_{+}-K_{+}^{*}\right) \pi^{2}} \\
& +\frac{\left\{4 W W^{*} \alpha^{2}+2 H H^{*}\left(K_{+}^{*}-2 \alpha^{2}\right)-K_{+}^{* 2}\left(W+W^{*}+\alpha^{2}\right)+2 K_{+}^{*}\left[W W^{*}+\left(W+W^{*}\right) \alpha^{2}\right]\right\} \ln \left(-K_{+}^{*}\right)}{\left(K_{-}-K_{+}^{*}\right)\left(-K_{-}^{*}+K_{+}^{*}\right)\left(-K_{+}+K_{+}^{*}\right) \pi^{2}} \\
& -\frac{-2 H^{2}+\left(K_{-}+K_{+}-2 W\right) W}{\left(K_{-}-K_{+}\right)^{2} \pi^{2}}-\frac{\left[\left(K_{-}+K_{+}\right)\left(4 H^{2}+K_{-} K_{+}\right)-8 K_{-} K_{+} W-4\left(K_{-}+K_{+}\right) W^{2}\right] \alpha^{2}}{2 K_{-}\left(K_{-}-K_{+}\right)^{2} K_{+} \pi^{2}} \\
& -\left[\ln \left(-K_{-}\right)-\ln \left(-K_{+}\right)\right] \frac{W\left[-2 K_{-} K_{+}+\left(K_{-}+K_{+}\right) W\right]+\left[-K_{-} K_{+}+2\left(K_{-}+K_{+}\right) W+4 W^{2}\right] \alpha^{2}+H^{2}\left(K_{-}+K_{+}-4 \alpha^{2}\right)}{\left(K_{-}-K_{+}\right)^{3} \pi^{2}} \\
& -\frac{-2 H^{* 2}+\left(K_{-}^{*}+K_{+}^{*}-2 W^{*}\right) W^{*}}{\left(K_{-}^{*}-K_{+}^{*}\right)^{2} \pi^{2}}-\frac{\left[\left(K_{-}^{*}+K_{+}^{*}\right)\left(4 H^{* 2}+K_{-}^{*} K_{+}^{*}\right)-8 K_{-}^{*} K_{+}^{*} W^{*}-4\left(K_{-}^{*}+K_{+}^{*}\right) W^{* 2}\right] \alpha^{2}}{2 K_{-}^{*}\left(K_{-}^{*}-K_{+}^{*}\right)^{2} K_{+}^{*} \pi^{2}} \\
& -\left[\ln \left(-K_{-}^{*}\right)-\ln \left(-K_{+}^{*}\right)\right] \\
& \times \frac{W^{*}\left[-2 K_{-}^{*} K_{+}^{*}+\left(K_{-}^{*}+K_{+}^{*}\right) W^{*}\right]+\left[-K_{-}^{*} K_{+}^{*}+2\left(K_{-}^{*}+K_{+}^{*}\right) W^{*}+4 W^{* 2}\right] \alpha^{2}+H^{* 2}\left(K_{-}^{*}+K_{+}^{*}-4 \alpha^{2}\right)}{\left(K_{-}^{*}-K_{+}^{*}\right)^{3} \pi^{2}}, \\
& \frac{-}{\pi^{2}}
\end{aligned}
$$



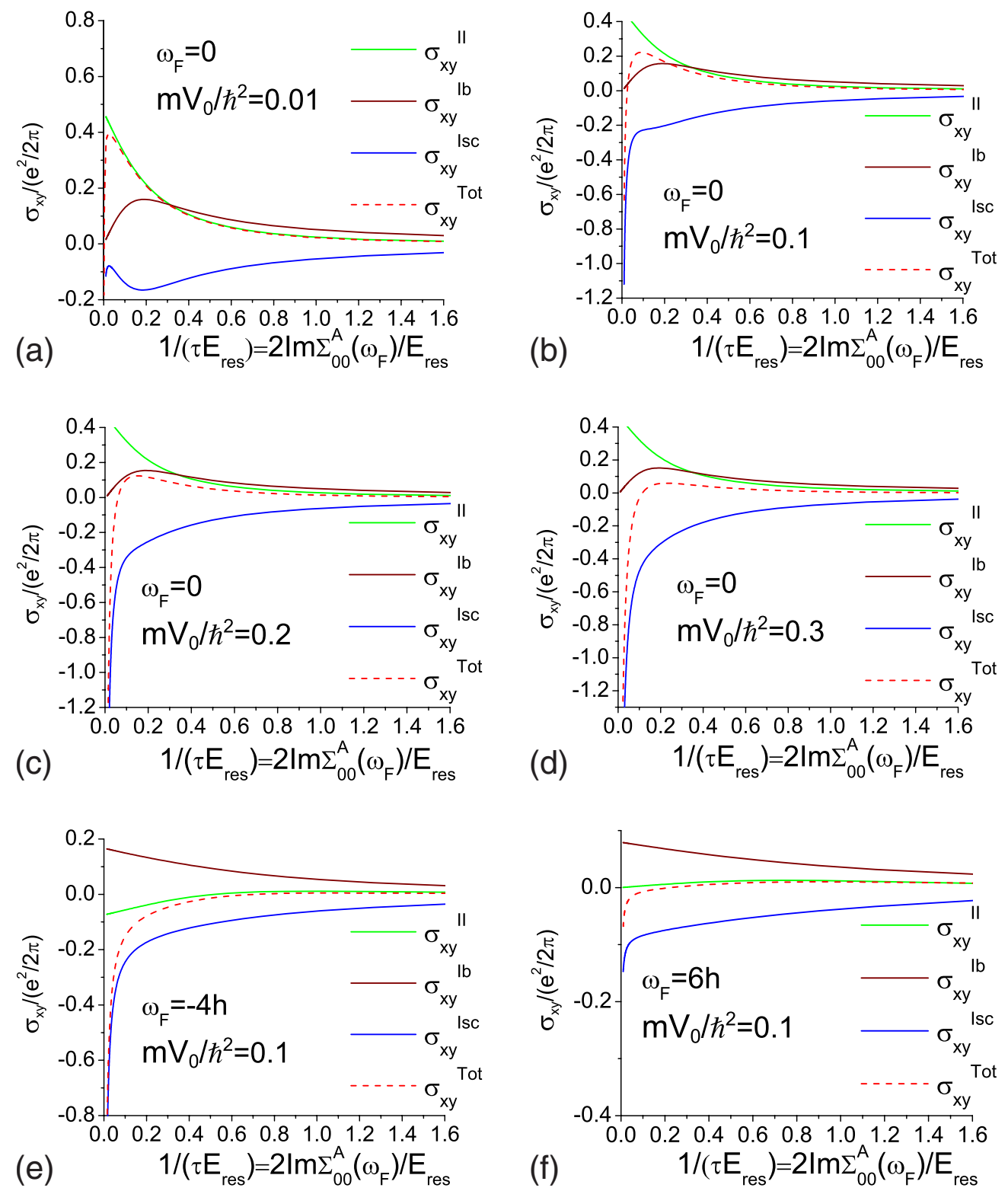

FIG. 13. (Color online) The anomalous Hall conductivity $\sigma_{x y}^{T o t}=\sigma_{x y}^{I b}+\sigma_{x y}^{I s c}+\sigma_{x y}^{I I}$ and its components $\left(\sigma_{x y}^{I b}, \sigma_{x y}^{I s c}, \sigma_{x y}^{I I}\right)$ vs the averaged relaxation rate $1 / \tau=2 \operatorname{Im} \Sigma_{00}^{A}$ (defined in Appendix B). The spin-orbit interaction strength is $2 m \alpha^{2} / E_{\text {res }}=3.59\left(E_{\text {res }}=10 h\right)$; the strength of impurities $V_{0}=0.01,0.1,0.2,0.3$; the Fermi energy $\varepsilon_{F} / E_{\mathrm{res}}=0.9$ for $\omega_{F}=0, \varepsilon_{F} / E_{\mathrm{res}}=0.5$ for $\omega_{F}=-4 h$, and $\varepsilon_{F} / E_{\mathrm{res}}=1.5$ for $\omega_{F}=6 h$. The Fermi energy $\omega_{F}$ corresponds here to the clean system and it is renormalized according to Eq. (35) in the presence of disorder. Dimensionality of quantities displayed in this plot is restored.

The analytical expressions for the self-consistent contributions to the conductivities $\sigma_{x y}^{I s c}$ and $\sigma_{y y}^{I s c}$ become

$$
\begin{aligned}
\frac{\sigma_{x y}^{I s c}}{e^{2} / \hbar}= & \frac{\alpha n_{i}\left\{\rho_{+-} T_{++} T_{--}^{*}\left[K_{-}^{* 2}+4(H+W)\left(H^{*}-W^{*}\right)\right]-\rho_{-+} T_{--} T_{++}^{*}\left[K_{-}^{* 2}+4(H-W)\left(H^{*}+W^{*}\right)\right]\right\} \ln \left(-K_{-}^{*}\right)}{2\left(K_{-}-K_{-}^{*}\right)\left(K_{-}^{*}-K_{+}\right)\left(K_{-}^{*}-K_{+}^{*}\right) \pi^{2} e E_{y}} \\
& -\frac{\alpha n_{i}\left\{\rho_{+-} T_{++} T_{--}^{*}\left[K_{-}^{2}+4(H+W)\left(H^{*}-W^{*}\right)\right]-\rho_{-+} T_{--} T_{++}^{*}\left[K_{-}^{2}+4(H-W)\left(H^{*}+W^{*}\right)\right]\right\} \ln \left(-K_{-}\right)}{2\left(K_{-}-K_{-}^{*}\right)\left(K_{-}-K_{+}\right)\left(K_{-}-K_{+}^{*}\right) \pi^{2} e E_{y}} \\
& +\frac{\alpha n_{i}\left\{\rho_{+-} T_{++} T_{--}^{*}\left[K_{+}^{* 2}+4(H+W)\left(H^{*}-W^{*}\right)\right]-\rho_{-+} T_{--} T_{++}^{*}\left[K_{+}^{* 2}+4(H-W)\left(H^{*}+W^{*}\right)\right]\right\} \ln \left(-K_{+}^{*}\right)}{2\left(K_{-}-K_{+}^{*}\right)\left(-K_{-}^{*}+K_{+}^{*}\right)\left(-K_{+}+K_{+}^{*}\right) \pi^{2} e E_{y}} \\
& -\frac{\alpha n_{i}\left\{\rho_{+-} T_{++} T_{--}^{*}\left[K_{+}^{2}+4(H+W)\left(H^{*}-W^{*}\right)\right]-\rho_{-+} T_{--} T_{++}^{*}\left[K_{+}^{2}+4(H-W)\left(H^{*}+W^{*}\right)\right]\right\} \ln \left(-K_{+}\right)}{2\left(K_{-}-K_{+}\right)\left(K_{-}^{*}-K_{+}\right)\left(K_{+}-K_{+}^{*}\right) \pi^{2} e E_{y}},
\end{aligned}
$$



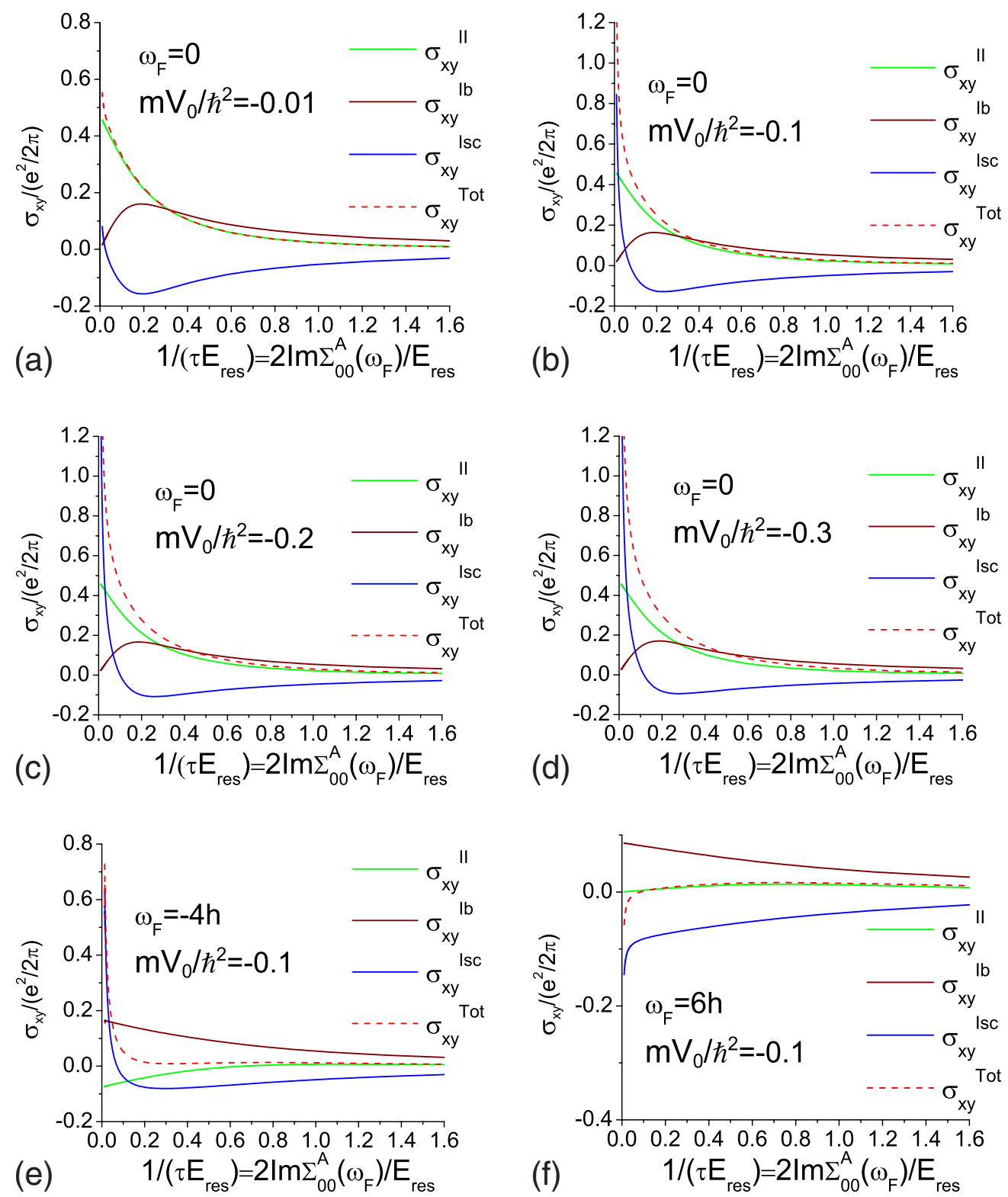

FIG. 14. (Color online) Identical to Fig. 13 plot with attractive disorder $\left(V_{0}=-0.01,-0.1,-0.2\right.$, and -0.3$)$.

$$
\begin{aligned}
\frac{\sigma_{y y}^{I s c}}{e^{2} / \hbar}= & \frac{i \alpha n_{i}\left\{\rho_{+-} T_{++} T_{--}^{*}\left[K_{-}^{2}+4(H+W)\left(H^{*}-W^{*}\right)\right]+\rho_{-+} T_{--} T_{++}^{*}\left[K_{-}^{2}+4(H-W)\left(H^{*}+W^{*}\right)\right]\right\} \ln \left(-K_{-}\right)}{2\left(K_{-}-K_{-}^{*}\right)\left(K_{-}-K_{+}\right)\left(K_{-}-K_{+}^{*}\right) \pi^{2} e E_{y}} \\
& -\frac{i \alpha n_{i}\left\{\rho_{+-} T_{++} T_{--}^{*}\left[K_{-}^{* 2}+4(H+W)\left(H^{*}-W^{*}\right)\right]+\rho_{-+} T_{--} T_{++}^{*}\left[K_{-}^{* 2}+4(H-W)\left(H^{*}+W^{*}\right)\right]\right\} \ln \left(-K_{-}^{*}\right)}{2\left(K_{-}-K_{-}^{*}\right)\left(K_{-}^{*}-K_{+}\right)\left(K_{-}^{*}-K_{+}^{*}\right) \pi^{2} e E_{y}} \\
& +\frac{i \alpha n_{i}\left\{\rho_{+-} T_{++} T_{--}^{*}\left[K_{+}^{2}+4(H+W)\left(H^{*}-W^{*}\right)\right]+\rho_{-+} T_{--} T_{++}^{*}\left[K_{+}^{2}+4(H-W)\left(H_{-}^{*}-K_{+}\right)\left(K_{+}-K_{+}^{*}\right)\right]\right\} \ln \left(-K_{+}\right)}{2\left(K_{-} e E_{y}\right.} \\
& -\frac{i \alpha n_{i}\left\{\rho_{+-} T_{++} T_{--}^{*}\left[K_{+}^{* 2}+4(H+W)\left(H^{*}-W_{-}^{*}\right)\right]+\rho_{-+} T_{--} T_{++}^{*}\left[K_{+}^{*}\right)\left(-K_{+}+K_{+}^{*}\right) \pi^{2} e E_{y}\right.}{\left.\left.2(H-W)\left(H^{*}+W^{*}\right)\right]\right\} \ln \left(-K_{+}^{*}\right)},
\end{aligned}
$$

where again all parameters are calculated at the Fermi surface and $T_{ \pm \pm}$and $\rho_{ \pm \mp}$ correspond to the elements of the matrices $\hat{T}_{e q}^{R}\left(\omega_{F}\right)$ and $\hat{\rho}\left(\omega_{F}\right)$, respectively.

\section{APPENDIX F: CALCULATION OF THE FERMI SEA CONDUCTIVITY}

For the electric field $\mathbf{E}$ along the $y$ axis $\mathbf{E}=\left(0, E_{y}\right)$, we perform momentum integrations in Eq. (43) arriving at the following expressions for conductivities $\sigma_{x y}^{I I}$ and $\sigma_{y y}^{I I}$ : 


$$
\begin{gathered}
\sigma_{x y}^{I I}=\sigma_{x y}^{I I b}+\sigma_{x y}^{I I s c}, \\
\sigma_{y y}^{I I}=0, \\
\frac{\sigma_{x y}^{I I b}}{e^{2} / \hbar}=-\int d \omega n_{F}\left\{\frac{4 i \alpha^{2}\left[H\left(1-\partial_{\omega} \Sigma_{00}^{R}\right)\left(K_{-}+K_{+}\right)+\partial_{\omega} \Sigma_{0 z}^{R}\left[K_{+} W+K_{-}\left(K_{+}+W\right)\right]\right]}{K_{-} K_{+}\left(K_{-}-K_{+}\right)^{2} \pi^{2}}\right. \\
\left.-\frac{2 i \alpha^{2}\left[4\left(1-\partial_{\omega} \Sigma_{00}^{R}\right) H+\partial_{\omega} \Sigma_{0 z}^{R}\left(K_{-}+K_{+}+4 W\right)\right]\left[\ln \left(-K_{-}\right)-\ln \left(-K_{+}\right)\right]}{\left(K_{-}-K_{+}\right)^{3} \pi^{2}}\right\}+ \text { c.c. }, \\
\frac{\alpha\left(\rho_{E_{y}-+}^{R}-\rho_{E_{y}+-}^{R}\right)\left(4 H^{2}+K_{-} K_{+}-4 W^{2}\right)\left(K_{-}^{2}-K_{+}^{2}+2 K_{-} K_{+}\left[-\ln \left(-K_{-}\right)+\ln \left(-K_{+}\right)\right]\right)}{2 K_{-} K_{+}\left(K_{+}-K_{-}\right)^{3} \pi^{2} /\left(T_{--} T_{++}\right)}+\text {c.c. }=0,
\end{gathered}
$$

where $T_{ \pm \pm}$corresponds to the elements of the matrix $\hat{T}_{e q}^{R}(\omega)$. The fact that $\sigma_{x y}^{I I s c}=0$ follows from the identity $4 H^{2}+K_{-} K_{+}$ $-4 W^{2} \equiv 0$.

As one can see, $\sigma_{y y}^{I I}$ and $\sigma_{x y}^{I I s c}$ contributions to the Fermi sea Hall conductivity vanish and the nonvanishing contribution $\sigma_{x y}^{I I b}$ depends on the self-consistently calculated $\Sigma_{00}^{R}(\omega)$ and $\Sigma_{0 z}^{R}(\omega)$ that are functions of energy. Calculation of $\sigma_{x y}^{I I b}$ from Eq. (F3) requires numerical integration over $\omega$.

\section{APPENDIX G: DETAILED RESULTS FOR THE HALL CONDUCTIVITY}

In order to gain more insight into the behavior of the anomalous Hall effect, in Figs. 13 and 14 we plot different components of the AHE conductivity, particularly the Fermi sea contribution $\sigma_{x y}^{I I}$, the bare bubble contribution $\sigma_{x y}^{I b}$ (this corresponds to $\sigma_{x y}^{\text {lint }}$ in Ref. 1), and the self-consistent contribution $\sigma_{x y}^{I s c}$ (this corresponds to $\sigma_{x y}^{\mathrm{ext}}$ in Ref. 1). In Fig. 13, we take the same parameters as in Figs. 7 and 8 of Ref. 1 and we find disagreement with Ref. 1 in the results for the contribution $\sigma_{x y}^{\text {ext }}\left(\sigma_{x y}^{I s c}\right)$. The contributions $\sigma_{x y}^{I b}$ and $\sigma_{x y}^{I I}$ perfectly agree with Ref. 1 .

In the clean limit $\tau \rightarrow \infty$, we see that $\sigma_{x y}^{I s c}$ and thus the total Hall conductivity $\sigma_{x y}^{T o t}$ diverge. This divergence $\left[\sigma_{x y}^{I s c}\right.$ $\sim 1 /\left(n_{i} V_{0}\right)$ in regions (ii) and (iii) and $\sigma_{x y}^{I s c} \sim 1 / n_{i}$ in region (i), see Fig. 2] is due to the skew scattering. The conductivity $\sigma_{x y}^{I S c}$ also contains the side-jump contribution which can be best seen in Fig. 13(a) in the sharp peak in the $\sigma_{x y}^{I s c}$ conductivity for small $1 / \tau$. The skew-scattering contribution decays much faster compared to the side-jump and/or intrinsic mechanisms as we go to larger $1 / \tau$. As a result, we can expect a crossover between the region dominated by the skew scattering and the region dominated by the side-jumpintrinsic mechanisms. When both subbands are partially occupied [see Figs. 13(f) and 14(f)], the higher order skew scattering is still present. However, we do not expect a wellpronounced crossover as the intrinsic contribution cancels the side-jump contribution in the metallic regime [see Eq. (45)]. By comparing Figs. 13(f) and 14(f), one can see that the higher order skew scattering (hybrid skew scattering) (Ref. 24) does not change sign when we change the sign of impurities.

When the side-jump-intrinsic and the skew-scattering components have opposite signs, as in Fig. 13, we observe the AHE sign change instead of the crossover. In Figs. 13(a)-13(d), the skew scattering is negative in the clean limit while the side-jump-intrinsic part is positive. This inevitably leads to the sign change of the conductivity $\sigma_{x y}$ as we increase the disorder.
${ }^{1}$ S. Onoda, N. Sugimoto, and N. Nagaosa, Phys. Rev. B 77, 165103 (2008).

${ }^{2}$ R. Shindou and L. Balents, Phys. Rev. B 77, 035110 (2008).

${ }^{3}$ E. H. Hall, Philos. Mag. 10, 301 (1880).

${ }^{4}$ W. Thomson, Proc. R. Soc. London 8, 546 (1856).

${ }^{5}$ R. Karplus and J. M. Luttinger, Phys. Rev. 95, 1154 (1954).

${ }^{6}$ Y. Taguchi, Y. Oohara, H. Yoshizawa, N. Nagaosa, and Y. Tokura, Science 291, 2573 (2001).

${ }^{7}$ T. Jungwirth, Q. Niu, and A. H. MacDonald, Phys. Rev. Lett. 88, 207208 (2002).

${ }^{8}$ M. Onoda and N. Nagaosa, J. Phys. Soc. Jpn. 71, 19 (2002).

${ }^{9}$ Y. Yao, L. Kleinman, A. H. MacDonald, J. Sinova, T. Jungwirth,
D.-S. Wang, E. Wang, and Q. Niu, Phys. Rev. Lett. 92, 037204 (2004).

${ }^{10}$ W.-L. Lee, S. Watauchi, V. L. Miller, R. J. Cava, and N. P. Ong, Science 303, 1647 (2004).

${ }^{11}$ C. Zeng, Y. Yao, Q. Niu, and H. H. Weitering, Phys. Rev. Lett. 96, 037204 (2006).

${ }^{12}$ E. I. Rashba, Semiconductors 42, 905 (2008); arXiv:0804.4181 (unpublished).

${ }^{13}$ G. Sundaram and Q. Niu, Phys. Rev. B 59, 14915 (1999).

${ }^{14}$ J. Sinova, T. Jungwirth, and J. Černe, Int. J. Mod. Phys. B 18, 1083 (2004).

${ }^{15}$ D. Culcer, A. MacDonald, and Q. Niu, Phys. Rev. B 68, 045327 
(2003).

${ }^{16}$ V. K. Dugaev, P. Bruno, M. Taillefumier, B. Canals, and C. Lacroix, Phys. Rev. B 71, 224423 (2005).

${ }^{17}$ N. A. Sinitsyn, Q. Niu, J. Sinova, and K. Nomura, Phys. Rev. B 72, 045346 (2005).

${ }^{18}$ S. Y. Liu, N. J. M. Horing, and X. L. Lei, Phys. Rev. B 74, 165316 (2006).

${ }^{19}$ J.-I. Inoue, T. Kato, Y. Ishikawa, H. Itoh, G. E. W. Bauer, and L. W. Molenkamp, Phys. Rev. Lett. 97, 046604 (2006).

${ }^{20}$ S. Onoda, N. Sugimoto, and N. Nagaosa, Phys. Rev. Lett. 97, 126602 (2006).

${ }^{21}$ M. F. Borunda, T. S. Nunner, T. Luck, N. A. Sinitsyn, C. Timm, J. Wunderlich, T. Jungwirth, A. H. MacDonald, and J. Sinova, Phys. Rev. Lett. 99, 066604 (2007).

${ }^{22}$ T. S. Nunner et al., Phys. Rev. B 76, 235312 (2007).

${ }^{23}$ T. Kato, Y. Ishikawa, H. Itoh, and J.-i. Inoue, New J. Phys. 9, 350 (2007)

${ }^{24}$ A. A. Kovalev, K. Vyborny, and J. Sinova, Phys. Rev. B 78, 041305(R) (2008).

${ }^{25}$ J. Smit, Physica (Amsterdam) 21, 877 (1955).

${ }^{26}$ P. Nozieres and C. Lewiner, J. Phys. (France) 34, 901 (1973).

${ }^{27}$ N. A. Sinitsyn, Q. Niu, and A. H. MacDonald, Phys. Rev. B 73, 075318 (2006).

${ }^{28}$ J. Smit, Physica (Amsterdam) 17, 612 (1951).

${ }^{29}$ L. Berger, Physica (Amsterdam) 30, 1141 (1964).

${ }^{30}$ T. McGuire and R. Potter, IEEE Trans. Magn. 11, 1018 (1975).

${ }^{31}$ O. Jaoul, I. A. Campbell, and A. Fert, J. Magn. Magn. Mater. 5, 23 (1977).

${ }^{32}$ J. Banhart and H. Ebert, Europhys. Lett. 32, 517 (1995).

${ }^{33}$ J. Velev, R. F. Sabirianov, S. S. Jaswal, and E. Y. Tsymbal, Phys. Rev. Lett. 94, 127203 (2005).

${ }^{34}$ A. W. Rushforth et al., Phys. Rev. Lett. 99, 147207 (2007).

${ }^{35}$ K. Vyborny, A. A. Kovalev, J. Sinova, and T. Jungwirth, Phys. Rev. B 79, 045427 (2009).

${ }^{36}$ T. Kato, Y. Ishikawa, H. Itoh, and J.-I. Inoue, Phys. Rev. B 77, 233404 (2008).

${ }^{37}$ K. Ueno, T. Fukumura, H. Toyosaki, M. Nakano, and M. Ka- wasaki, Appl. Phys. Lett. 90, 072103 (2007).

${ }^{38}$ T. Miyasato, N. Abe, T. Fujii, A. Asamitsu, S. Onoda, Y. Onose, N. Nagaosa, and Y. Tokura, Phys. Rev. Lett. 99, 086602 (2007).

${ }^{39}$ T. Fukumura, H. Toyosaki, K. Ueno, M. Nakano, T. Yamasaki, and M. Kawasaki, Jpn. J. Appl. Phys., Part 2 46, L642 (2007).

${ }^{40}$ D. Venkateshvaran, W. Kaiser, A. Boger, M. Althammer, M. S. Ramachandra Rao, S. T. B. Goennenwein, M. Opel, and R. Gross, Phys. Rev. B 78, 092405 (2008).

${ }^{41}$ A. Fernandez-Pacheco, J. M. De Teresa, J. Orna, L. Morellon, P. A. Algarabel, J. A. Pardo, and M. R. Ibarra, Phys. Rev. B 77, 100403(R) (2008).

${ }^{42}$ J. Rammer and H. Smith, Rev. Mod. Phys. 58, 323 (1986).

${ }^{43}$ G. D. Mahan, Many-Particle Physics (Plenum, New York, 1990).

${ }^{44}$ P. Streda, J. Phys. C 15, L717 (1982).

${ }^{45}$ V. K. Dugaev, A. Crépieux, and P. Bruno, Phys. Rev. B 64, 104411 (2001)

${ }^{46}$ N. A. Sinitsyn, A. H. MacDonald, T. Jungwirth, V. K. Dugaev, and J. Sinova, Phys. Rev. B 75, 045315 (2007).

${ }^{47}$ G. E. Pikus and A. N. Titkov, Optical Orientation (NorthHolland, Amsterdam, 1984).

${ }^{48}$ E. Rashba and V. Sheka, Landau Level Spectroscopy (NorthHolland, Amsterdam, 1991), p. 167.

${ }^{49}$ X. C. Zhang, A. Pfeuffer-Jeschke, K. Ortner, V. Hock, H. Buhmann, C. R. Becker, and G. Landwehr, Phys. Rev. B 63, 245305 (2001).

${ }^{50}$ H.-A. Engel, B. I. Halperin, and E. I. Rashba, Phys. Rev. Lett. 95, 166605 (2005).

${ }^{51}$ D. J. Thouless, M. Kohmoto, M. P. Nightingale, and M. den Nijs, Phys. Rev. Lett. 49, 405 (1982).

${ }^{52}$ G. Mihály, M. Csontos, S. Bordács, I. Kézsmárki, T. Wojtowicz, X. Liu, B. Jankó, and J. K. Furdyna, Phys. Rev. Lett. 100, 107201 (2008).

${ }^{53}$ J. Schliemann and D. Loss, Phys. Rev. B 68, 165311 (2003).

${ }^{54}$ T. S. Nunner, G. Zaránd, and F. von Oppen, Phys. Rev. Lett. 100, 236602 (2008).

${ }^{55}$ A. W. Rushforth et al., J. Magn. Magn. Mater. 321, 1001 (2009). 